\title{
Meromorphic Zeta Functions for Analytic Flows
}

\author{
David Fried ${ }^{\star}$ \\ Department of Mathematics, Boston University, Boston, MA 02215, USA
}

Received: 12 August 1994/in revised form: 3 January 1995

Dedicated to Steve Smale

\begin{abstract}
We extend to hyperbolic flows in all dimensions Rugh's results on the meromorphic continuation of dynamical zeta functions. In particular we show that the Ruelle zeta function of a negatively curved real analytic manifold extends to a meromorphic function on the complex plane.
\end{abstract}

In this paper we address a problem Smale poses in his survey article on dynamical systems ([Sm], II.4): given an isolated, compact, hyperbolic set $\Omega$ for a flow $\phi$ on a manifold $M$, find a meromorphic function on $\mathbf{C}$ which admits the product expansion

$$
R(z)=\prod_{\gamma}\left(1-e^{-z /(\gamma)}\right)
$$

for $\operatorname{Re} z \gg 0$, where $\gamma$ runs over the periodic trajectories in $\Omega$ of multiplicity 1 and $\ell(\gamma)$ denotes the period of $\gamma$. The theorem in Sect. 7 does this for $\phi$ a $C^{\omega}$ (real analytic) flow.

In fact we meromorphically extend any "zeta function in one variable for $(\Omega, \phi)$ " to $\mathbf{C}$. A precise definition is given at the end of Sect. 6, with a discussion which shows how it includes all the usual examples. In particular we treat Selberg's zeta function $S(z)$ for a cocompact Fuchsian group $\Gamma$, where $\Omega=\Gamma \backslash P S l(2, \mathbf{R})$ and $\phi_{t}$ is given by right multiplication by the one parameter group diag $\left(e^{t / 2}, e^{-t / 2}\right)$, and where $S(z)$ is defined as

$$
S(z)=R(z) R(z+1) R(z+2) \ldots
$$

In [Se], Selberg uses his trace formula to meromorphically extend $S(z)$. Hence $R(z)=S(z) / S(z+1)$ also has a meromorphic extension in this case, which motivated Smale's problem.

This paper combines the methods of a seminal paper of Ruelle with an innovative idea of Rugh. Ruelle's paper [R1] concerns the case where the stable and unstable bundles $E^{s}, E^{u}$ of $\phi_{t} \mid \Omega$ extend to $C^{\omega}$ bundles on a neighborhood of $\Omega$.

$\star$ This work was supported in part by I.H.E.S. and the National Science Foundation. 
He employs the Markov partitions of Sinai and Bowen and a combinatorial argument of Manning and Bowen to produce a finite sequence of trace class operators $L_{m}(z), m=0,1,2, \ldots, z \in \mathbf{C}$, such that

$$
\prod_{m \geqq 0} \operatorname{det}\left(I-L_{m}(z)\right)^{(-1)^{m}}=\exp -\sum_{\gamma} \frac{1}{\mu(\gamma)} \frac{1}{\operatorname{det}\left(I-S_{\gamma}\right)} e^{-z \ell(\gamma)}, \quad \operatorname{Re} z \gg 0,
$$

where $\gamma$ runs over all periodic trajectories in $\Omega, S_{\gamma}$ is the stable summand of the linear Poincare map of $\gamma$ and $\mu(\gamma)$ is the multiplicity of $\gamma$. Ruelle's transfer operators $L_{m}(z)$ are defined by the action of real analytic contraction maps on spaces of holomorphic functions associated to the partition. This product formula furnishes the meromorphic extension of the right-hand side, which we define to be the Selberg function of $(\Omega, \phi)$. For $(\Omega, \phi)$ as in the preceding paragraph, $S_{\gamma}=e^{-\ell(\gamma)}$, $1 / \operatorname{det}\left(I-S_{\gamma}\right)=\sum_{k=0}^{\infty} e^{-k \ell(\gamma)}$, and $\exp -\sum_{\gamma} \frac{1}{\mu(\gamma)} e^{-(z+k) \ell(\gamma)}=R(z+k)$ so the Selberg function is just $S(z)$. In this way Ruelle gives a dynamical construction of the meromorphic extension of $S(z)$, independent of Selberg's trace formula.

Unfortunately the analyticity condition on $E^{s}, E^{u}$ holds in rather few examples. For instance, our theorem applies to the geodesic flow on the unit tangent bundle $\Omega=U Q$ of any connected, closed $C^{\omega}$ Riemannian manifold $Q$ of negative curvature. These bundles are only known to be $C^{\omega}$ when $Q$ is locally symmetric, that is the quotient of $F$-hyperbolic $n$-space by a discrete group of isometries, with $n \geqq 2$, $F=\mathbf{R}, \mathbf{C}, \mathbf{H}$ or $n=2, F=\mathbf{O}$. For $Q$ of dimension 2 or 3 the only known examples with $E^{s}, E^{u}$ analytic have constant sectional curvature.

A way around a similar problem is due to Rugh. Given a hyperbolic analytic map on a rectangle $I_{1} \times I_{2}$, he introduces a space of holomorphic functions of 2 variables, one in a neighborhood of $I_{2}$ in $\mathbf{C}$, and the other in the exterior of a neighborhood of $I_{1}$ in $\mathbf{C}$ and a corresponding transfer operator [Ru]. Since the points of geometric interest lie outside the domain of these functions, the significance of the eigenfunctions of his transfer operator is obscured.

Recall, however, that there is a pairing between forms $f(z) d z$ holomorphic on the disc $|z| \leqq 1$ and functions $g(z)$ holomorphic on the disc $|z| \geqq 1$ in the Riemann sphere $\mathbf{C P}^{1}$, given by

$$
\langle g(z) \mid f(z) d z\rangle=\frac{1}{2 \pi i} \oint g(z) f(z) d z .
$$

For $i \geqq 0, j \geqq 0$ we have $\left\langle z^{-j-1} \mid z^{i} d z\right\rangle=\delta_{l j}$. This shows that Rugh's function space combines a function space in one variable with part of the dual of a space of holomorphic forms in the other variable.

In Sect. 2 we consider 2 regions (closures of bounded open sets) in complex space $W \subset \mathbf{C}^{u}$ and $Z \subset \mathbf{C}^{s}$. We define $A(Z)$ as the uniform limits of polynomials on $Z$ and $V(W)$ as the forms $\phi(w) d w_{1} \wedge \cdots \wedge d w_{u}$ with $\phi(w) \in A(W)$. Then we define $K(W, Z)$ as the Banach space of linear operators from $V(W)$ to $A(Z)$ that are norm limits of finite rank operators. This space of "kernels" replaces the holomorphic function spaces of Ruelle and Rugh.

In Sect. 1 we formalize the notion of hyperbolic correspondence $f$ by parametrizing the correspondence as the graph of a certain cross map c. If $f$ goes from $X \times Y$ to $X^{\prime} \times Y^{\prime}, c$ takes $X^{\prime} \times Y$ to $X \times Y^{\prime}$. For $f$ hyperbolic, $c$ is a contraction. Passing to small complex neighborhoods, we extend a $C^{\omega} c$ in Sect. 2 to a holomorphic contraction $C: W^{\prime} \times Z \rightarrow W \times Z^{\prime}$ that is the cross map of a hyperbolic correspondence $F$ from $W \times Z$ to $W^{\prime} \times Z^{\prime}$. Then $F$ defines a kernel transfer $L_{F}: K\left(W^{\prime}, Z^{\prime}\right) \rightarrow K(W, Z)$. 
Given $r$, a $d \times d$ matrix over $K\left(W^{\prime}, Z\right)$, there is an analogous operator $L_{r, F}$ on $d$-tuples of kernels. In the split case, where $C$ is the Cartesian product of a contraction $C_{1}: W^{\prime} \rightarrow W$ and a contraction $C_{2}: Z \rightarrow Z^{\prime}, L_{r, F}$ pulls back a $d$-tuple of kernels to $K\left(W^{\prime}, Z\right)$ using $C_{2}$, multiplies it by $r$, and then pushes this $d$-tuple of kernels forward to $K(W, Z)$ using $C_{1}$. In general, $L_{r, F}$ is constructed as the partial adjoint in the first factor of an operator on $d$-tuples of holomorphic forms that involves pullback by $C$. $L_{r, F}$ is well approximated in norm by finite rank operators, hence of trace class.

These linear operators are dual to the nonlinear graph transform operators of hyperbolic dynamics. For provided we identify $V(W)$ with $A(W)$, a function $f: W \rightarrow Z$ with $f(W) \subset \subset Z$ and $f$ a uniform limit of polynomials defines a linear functional on $K(W, Z)$ by $k \mapsto \operatorname{Tr}\left(k f^{*}\right)$, where $k f^{*}: A(Z) \rightarrow A(Z)$.

In Sect. 3 we show that kernel transfer behaves well under composition of correspondences. For $W^{\prime}=W$ and $Z^{\prime}=Z$, the trace of $L_{r, F}$ is calculated in Sect. 4. In these 2 sections we use the holomorphic fixed point formula of Atiyah-Bott instead of the explicit contour integrations of [Ru]. In Sect. 5 we calculate the Fredholm determinant $\operatorname{det}(I-L)$ for $L$ a block operator whose entries are of the form $L_{r, F}$, in terms of the periodic points of a finite system of hyperbolic correspondences.

Given a complex vector bundle $\xi$ over $\Omega$, the various lifts $\psi_{t}: \xi \rightarrow \xi$ of $\phi_{t} \mid \Omega$ to flows of bundle morphisms form a parameter space. For $\psi$ sufficiently contractive we use the trace $\chi_{\gamma}(\psi)$ of the holonomy of $\psi$ around closed orbits $\gamma$ to define the zeta function of this lift:

$$
\zeta(\psi)=\exp -\sum_{\gamma} \frac{1}{\mu(\gamma)} \frac{\chi_{\gamma}(\psi)}{\left|\operatorname{det}\left(I-\mathbf{P}_{\gamma}\right)\right|}=\exp -\int_{0}^{\infty} \frac{1}{t} d v(t),
$$

where $v=\operatorname{Tr}^{b} \psi_{t}^{*}$ is the flat-trace of the pullback operator $\psi_{t}^{*}$ on sections of $\xi$. Then we show that each example in the literature of a zeta function of the variable $z$ associated to $(\Omega, \phi)$ is a ratio of 2 functions of the special form $\zeta\left(\psi^{z a}\right)$. Here $\psi^{z a}$ is a curve in our parameter space, parametrized by $z \in \mathbf{C}$ :

$$
\psi_{t}^{z a}(v)=\exp \left[-z \int_{0}^{t} a\left(\psi_{s} v\right) d s\right] \psi_{t}(v)
$$

where $a$ is a given function on $\xi$, constant on fibers, with positive real part. For $a \equiv 1$, we define $\zeta\left(\psi^{z}\right)=T_{\psi}^{b}(z)$ to be the flat-trace function of the lift $\psi$. Taking the ratio of pairs of flat-trace functions, we obtain any Ruelle or Selberg function and also any of the torsion functions $Z_{\alpha}(z)$ that arise in Lefschetz formulas for $(\Omega, \phi)$.

We show in Sect. 7 that $\zeta\left(\psi^{z a}\right)$ is a finite alternating product of Fredholm determinants, where each factor $\operatorname{det} I-L_{m}(z)$ involves a holomorphic family $L_{m}(z)$ of operators associated to a system of holomorphic correspondences. This gives a meromorphic extension of $\zeta\left(\psi^{z a}\right)$ to $\mathbf{C}$.

In Sect. 7 we formulate 2 conjectures, one concerning Lefschetz formulas for geodesic flows on negatively curved manifolds and the other concerning the analyticity of $\zeta\left(\psi^{z a}\right)$.

Our combination of holomorphic function spaces and their duals is seen most clearly in a naive example, given as I. in Sect. 8, where we treat hyperbolic toral automorphisms without Markov partitions. The particular operators used in Sect. 5 are illustrated in Sect. 8, II, where we give a complete spectral analysis of a certain example with $u=s=1$. 
In Sect. 9 we study the length spectrum of a $C^{\omega}$ basic set in terms of the divisor of its Ruelle function. This leads to an asymptotic statement with a sharp error term for the averaged length distribution.

In Sect. 10 we derive a Lefschetz formula for a nonsingular Smale flow (i.e. an Axiom A-No Cycles $C^{\omega}$ flow with one-dimensional nonwandering set and no stationary points), relating the local properties of its closed orbits to the ambient topology. This takes the form of finding R-torsion as a special value of a certain zeta function.

Consider any $C^{\omega}$ metric $g$ of negative curvature on $\Gamma \backslash H^{2}$, where $\Gamma$ is a cocompact Fuchsian group. As in Sect. 6, the resulting geodesic flow $\phi$ on $\Gamma \backslash U H^{2}=$ $\Gamma \backslash P S l(2, \mathbf{R})$ has a Selberg function $S^{g}(z)$ that equals $S(z)$ for the metric $g_{0}$ induced from $H^{2}$. Since the unstable bundle for $g$ is not $C^{\omega}$ in general we cannot expect to meromorphically continue $S^{g}(z)$. However, we can write $S(z)=T_{\psi^{+}}^{b}(z) / T_{\psi^{-}}^{b}(z)$, where $\psi^{+}$and $\psi^{-}$are certain lifts, and this ratio defines a meromorphic function $\tilde{S}^{g}(z)$ on $\mathbf{C}$ for any $g$. The order of $\tilde{S}^{g}(z)$ is at most 3 , by the theorem in Sect. 7 . However Selberg showed that the order of $S(z)$ is 2 , and we seem to have an explosion of order when going from $S(z)=\tilde{S}^{g_{0}}(z)$ to $\tilde{S}^{g}(z)$. It would be interesting to verify this phenomenon numerically. We expect the instant creation of many nearby pairs of zeroes and poles for $g$ near $g_{0}$.

Since this paper was written, Rugh has extended his results to hyperbolic flows in dimension 3 [Ru2] and Kitaev has made progress on the case of nonanalytic diffeomorphisms [K].

We are pleased to dedicate this paper to Steve Smale, whose bold suggestions have stimulated a generation of researchers in dynamical systems.

\section{Contents:}

1. The iterates of a hyperbolic correspondence

2. The transfer operator for a $C^{\omega}$ hyperbolic correspondence

3. Functoriality of transfer

4. The trace of the kernel transfer

5. Zeta functions for a $C^{\omega}$ system of hyperbolic correspondences

6. Zeta functions for a hyperbolic set of a flow

7. Meromorphic extension of $T^{b}(z)$ and $\zeta(\psi)$

8. Examples

9. The explicit formula for a $C^{\omega}$ basic set

10. Lefschetz formulas for Smale flows

\section{Section 1. The Iterates of a Hyperbolic Correspondence}

Let $X, X^{\prime}, Y$ and $Y^{\prime}$ be complete metric spaces and $f \subset(X \times Y) \times\left(X^{\prime} \times Y^{\prime}\right)$ a closed correspondence from $X \times Y$ to $X^{\prime} \times Y^{\prime}$. We write $f(x, y)$ for the projection to $X^{\prime} \times Y^{\prime}$ of $f \cap\left(\{(x, y)\} \times\left(X^{\prime} \times Y^{\prime}\right)\right)$. If for each $x^{\prime} \in X^{\prime}$ and $y \in Y$ there is a unique $x \in X$ and $y^{\prime} \in Y$ with $\left(x^{\prime}, y^{\prime}\right) \in f(x, y)$, we say $f$ admits the cross map $c: X^{\prime} \times Y \rightarrow X \times Y^{\prime}$ with $c\left(x^{\prime}, y\right)=\left(x, y^{\prime}\right)$. Then this map $c$ determines the correspondence $f$ by the rule

$$
\left(x^{\prime}, y^{\prime}\right) \in f(x, y) \Leftrightarrow\left(x, y^{\prime}\right)=c\left(x^{\prime}, y\right) .
$$


Here the projection $f \rightarrow Y \times X^{\prime}$ is a homeomorphism and $f$ is parametrized by $\left(x^{\prime}, y\right) \mapsto\left(c_{1}\left(x^{\prime}, y\right), y, x^{\prime}, c_{2}\left(x^{\prime}, y\right)\right)$.

If the components $c_{1}: X^{\prime} \times Y \rightarrow X$ and $c_{2}: X^{\prime} \times Y \rightarrow Y^{\prime}$ are uniformly contracting in $x^{\prime}$ for $y$ fixed and in $y$ for $x^{\prime}$ fixed we say $f$ is a hyperbolic correspondence. In most applications, $f$ is (the graph of) a homeomorphism from a subset of $X \times Y$ to a subset of $X^{\prime} \times Y^{\prime}$ which expands in the first factor and contracts in the second. However we allow other situations, such as when $c$ is constant. In this case, if $\left(x_{0}, y_{0}^{\prime}\right)$ is the value of $c$ then

$$
f\left(x_{0}, y\right)=X^{\prime} \times\left\{y_{0}^{\prime}\right\}, \quad f(x, y)=\emptyset \quad \text { for } \quad x \neq x_{0} .
$$

This correspondence is the acme of hyperbolicity since it contracts infinitely in $y$ and "expands infinitely" in $x$.

In $[\mathrm{Ru}]$ the case where $X, X^{\prime}, Y$ and $Y^{\prime}$ are compact intervals and $c$ is real analytic was discussed. There the components $c_{1}$ and $c_{2}$ were called "pinning coordinates." There are advantages to combining them into one map, as will be clear in Sect. 2.

To find the inverse correspondence $f^{-1}$ from $X^{\prime} \times Y^{\prime}$ to $X \times Y$ we switch 2 pairs of coordinates in $f$, whereas the graph of $c$ is found by switching only 1 pair. Hence $c$ is a "partial inverse" of $f$. When $c_{1}$ depends on $x^{\prime}$ alone and $c_{2}$ depends on $y$ alone we say $f$ (or $c$ ) is split. In this case, $f(x, y)=c_{1}^{-1}\{x\} \times\left\{c_{2}(y)\right\}$ so $f$ is the Cartesian product of $c_{1}^{-1}$ and $c_{2}$.

The composition $h=g \circ f$ of 2 hyperbolic correspondences $f: X \times Y \rightarrow X^{\prime} \times$ $Y^{\prime}$ and $g: X^{\prime} \times Y^{\prime} \rightarrow X^{\prime \prime} \times Y^{\prime \prime}$ admits a cross map and is frequently a hyperbolic correspondence. Namely $\left(x^{\prime \prime}, y^{\prime \prime}\right) \in h(x, y)$ if for some $\left(x^{\prime}, y^{\prime}\right) \in X^{\prime} \times Y^{\prime}$,

$$
c\left(x^{\prime}, y\right)=\left(x, y^{\prime}\right) \text { and } d\left(x^{\prime \prime}, y^{\prime}\right)=\left(x^{\prime}, y^{\prime \prime}\right)
$$

where $c$ and $d$ are the cross maps for $f$ and $g$, respectively. Thus

$$
x^{\prime}=d_{1}\left(x^{\prime \prime}, c_{2}\left(x^{\prime}, y\right)\right) \text { and } y^{\prime}=c_{2}\left(d_{1}\left(x^{\prime \prime}, y^{\prime}\right), y\right) .
$$

Since $d_{1}\left(x^{\prime \prime},-\right)$ and $c_{2}(-, y)$ are contraction mappings for $x^{\prime \prime}$ and $y$ fixed, $x^{\prime}$ and $y^{\prime}$ are uniquely determined by these equations. On the other hand, as $X^{\prime}$ and $Y^{\prime}$ are complete we can use the contraction mapping theorem to define $x^{\prime}, y^{\prime}$ in terms of $x^{\prime \prime}, y$ by these equations. We obtain a cross map $e$ for $h$, with

$$
e\left(x^{\prime \prime}, y\right)=\left(c_{1}\left(x^{\prime}, y\right), d_{2}\left(x^{\prime \prime}, y^{\prime}\right)\right) \text {. }
$$

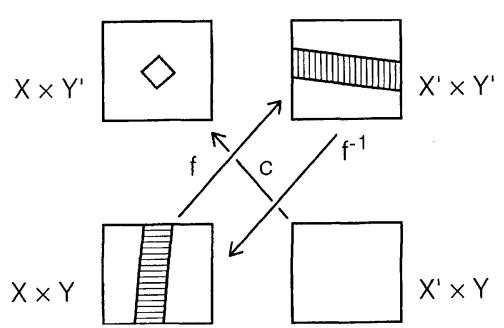

Fig. 1. A hyperbolic correspondence $f$ and its cross map $c$. 
To check whether $h$ is a hyperbolic correspondence, we introduce contraction constants $\alpha_{c}$ for $c_{1}(-, y), \beta_{c}$ for $c_{1}\left(x^{\prime},-\right), \gamma_{c}$ for $c_{2}(-, y)$ and $\delta_{c}$ for $c_{2}\left(x^{\prime},-\right)$, good for all $x^{\prime} \in X^{\prime}, y \in Y$. We refer to $\beta_{c}, \gamma_{c}$ as minor constants and $\alpha_{c}, \delta_{c}$ as major constants. Likewise we define contraction constants $\alpha_{d}, \beta_{d}, \gamma_{d}, \delta_{d}$ and Lipschitz constants $\alpha_{e}, \beta_{e}, \gamma_{e}, \delta_{e}$. We estimate the latter from above, as follows.

\section{Lemma 1.}

$$
\left(\begin{array}{ll}
\alpha_{e} & \beta_{e} \\
\gamma_{e} & \delta_{e}
\end{array}\right) \leqq\left(\begin{array}{cc}
\alpha_{c} \alpha_{d}\left(1-\beta_{d} \gamma_{c}\right)^{-1} & \beta_{c}+\alpha_{c} \beta_{d} \delta_{c}\left(1-\beta_{d} \gamma_{c}\right)^{-1} \\
\gamma_{d}+\alpha_{d} \gamma_{c} \delta_{d}\left(1-\beta_{d} \gamma_{c}\right)^{-1} & \delta_{c} \delta_{d}\left(1-\beta_{d} \gamma_{c}\right)^{-1}
\end{array}\right)
$$

Proof. We will bound $\gamma_{e}$, the other cases being handled similarly. Suppose $x^{\prime \prime}, x_{0}^{\prime \prime} \in$ $X^{\prime \prime}$ and $y \in Y$. Then

$$
\left|e_{2}(-, y)\right|_{x_{0}^{\prime \prime}}^{x^{\prime \prime}}|=| d_{2}\left(x^{\prime \prime}, y^{\prime}\right)-d_{2}\left(x_{0}^{\prime \prime}, y_{0}^{\prime}\right) \mid
$$

where $y^{\prime}$ is as above and $y_{0}^{\prime}=c_{2}\left(d_{1}\left(x_{0}^{\prime \prime}, y_{0}^{\prime}\right), y\right)$. (To simplify notation, we assume our metric spaces are isometrically embedded as subspaces of some normed vector space.) We have

$$
\begin{aligned}
\left|y^{\prime}-y_{0}^{\prime}\right| & \leqq \gamma_{c}\left|d_{1}\left(x^{\prime \prime}, y^{\prime}\right)-d_{1}\left(x_{0}^{\prime \prime}, y_{0}^{\prime}\right)\right| \\
& \leqq \gamma_{c}\left(\left|d_{1}\left(x^{\prime \prime}, y^{\prime}\right)-d_{1}\left(x^{\prime \prime}, y_{0}^{\prime}\right)\right|+\left|d_{1}\left(x^{\prime \prime}, y_{0}^{\prime}\right)-d_{1}\left(x_{0}^{\prime \prime}, y_{0}^{\prime}\right)\right|\right) \\
& \leqq \gamma_{c}\left(\beta_{d}\left|y^{\prime}-y_{0}^{\prime}\right|+\alpha_{d}\left|x^{\prime \prime}-x_{0}^{\prime \prime}\right|\right)
\end{aligned}
$$

so that

$$
\left|y^{\prime}-y_{0}^{\prime}\right| \leqq\left(1-\beta_{d} \gamma_{c}\right)^{-1} \alpha_{d} \gamma_{c}\left|x^{\prime \prime}-x_{0}^{\prime \prime}\right|
$$

Thus

$$
\begin{aligned}
\left|e_{2}(-, y)\right|_{x_{0}^{\prime \prime}}^{x^{\prime \prime}} \mid & \leqq\left|d_{2}\left(x^{\prime \prime}, y^{\prime}\right)-d_{2}\left(x_{0}^{\prime \prime}, y^{\prime}\right)\right|+\left|d_{2}\left(x_{0}^{\prime \prime}, y^{\prime}\right)-d_{2}\left(x_{0}^{\prime \prime}, y_{0}^{\prime}\right)\right| \\
& \leqq \gamma_{d}\left|x^{\prime \prime}-x_{0}^{\prime \prime}\right|+\delta_{d}\left|y^{\prime}-y_{0}^{\prime}\right|
\end{aligned}
$$

Using our bound on $\left|y^{\prime}-y_{0}^{\prime}\right|$ we obtain the desired estimate on $\gamma_{e}$.

Clearly $h$ is a hyperbolic correspondence whenever the 4 matrix entries on the right in this lemma are less than 1 . To apply this criterion to a composition of a large number of hyperbolic correspondences, we will suppose that they are nearly split as follows.

Suppose that for $i=0,1,2, \ldots$ we have complete metric spaces $X^{(i)}, Y^{(i)}$ and hyperbolic correspondences $f^{(i)}: X^{(i)} \times Y^{(i)} \rightarrow X^{(i+1)} \times Y^{(i+1)}$ with cross maps $C^{(i)}$ : $X^{(l+1)} \times Y^{(l)} \rightarrow X^{(l)} \times Y^{(l+1)}$ and contraction constants $\alpha, \beta, \gamma, \delta$ that are independent of $i$. Then if the minor constants $\beta$ and $\gamma$ are small compared to the major constants $\alpha$ and $\delta$, the following proposition asserts that each composition $f^{(m-1)} \circ \cdots \circ f^{(0)}$ will be hyperbolic. For motivation, note that the composition of split hyperbolic correspondences is obviously split hyperbolic and split is equivalent to $\beta=0=\gamma$.

Proposition 1. The composition of a sequence of hyperbolic correspondences is hyperbolic provided the major constants are bounded by some constant $\sigma<1$ and the minor constants are bounded by some $\rho<1$, with $\rho$ a positive function of $\sigma$. 
Proof. Let $\rho=\max \{\beta, \gamma\}, \sigma=\max \{\alpha, \delta\}$ and choose $r$ positive with $r^{2}<1-$ $\sigma$. We define $\rho_{l}, \sigma_{i}$ for $i \geqq 0$ by $\sigma_{0}=\sigma, \sigma_{i+1}=\sigma_{i}^{2}\left(1-r^{2}\right)^{-1}, \rho_{0}=\rho, \rho_{l+1}=$ $\rho_{i}\left(1+\sigma_{i+1}\right)$. Our choice of $r$ shows that $\sigma_{l}$ is a decreasing sequence and the series $\sum_{l} \sigma_{l}$ is summable. Clearly $\rho_{i}$ is an increasing sequence. Assuming

$$
\rho<r / \prod_{i=1}^{\infty}\left(1+\sigma_{l}\right)
$$

we find that $\rho_{i}<r$ for all $i$. In particular $\sigma_{i}<1$ and $\rho_{i}<1$ for all $i$.

Now we use $(*)$ to show by induction on $k=0,1,2, \ldots$ that any composition $f^{(i+m-1)} \circ \cdots \circ f^{(i)}, 1 \leqq m \leqq 2^{k}, i \geqq 0$ is hyperbolic, and that for $i \geqq 0$ and $2^{k-1} \leqq m \leqq 2^{k}$ the minor constants are no more than $\rho_{k}$ and the major constants are no more than $\sigma_{k}$. For $k=0$, this is our hypothesis on the $f^{(i)}$ combined with the definition of $\sigma_{0}, \rho_{0}$. Assume it holds for $k \geqq 0$. Then if $2^{k} \leqq$ $m \leqq 2^{k+1}$, we write $m=m_{1}+m_{2}$ with $2^{k-1} \leqq m_{1}, m_{2} \leqq 2^{k}$ and apply Lemma 1 to $g=f^{(i+m-1)} \circ \cdots \circ f^{\left(i+m_{2}\right)}$ and $f=f^{\left(l+m_{2}-1\right)} \circ \cdots \circ f^{(l)}$. Using the induction hypothesis to estimate the constants on the right-hand side of Lemma 1 gives

$$
\begin{gathered}
\alpha_{e}, \delta_{e} \leqq \sigma_{k}^{2}\left(1-\rho_{k}^{2}\right)^{-1}<\sigma_{k+1}, \\
\beta_{e}, \gamma_{e} \leqq \rho_{k}+\sigma_{k}^{2} \rho_{k}\left(1-\rho_{k}^{2}\right)<\rho_{k+1},
\end{gathered}
$$

since $\rho_{k}<r$. The proposition follows.

\section{Section 2. The Transfer Operator for a $C^{\omega}$ Hyperbolic Correspondence}

Let $u, s$ be nonnegative integers and let $X, X^{\prime}$ (respectively $Y, Y^{\prime}$ ) be regions in $\mathbf{R}^{u}$ (respectively $\mathbf{R}^{s}$ ), where by region we mean a compact set with dense interior. Suppose $f$ is a hyperbolic correspondence from $X \times Y$ to $X^{\prime} \times Y^{\prime}$ such that the cross map $c$ is real analytic, i.e. given by a convergent power series near every point of $X^{\prime} \times Y$. We will attach Banach spaces to $X \times Y$ and $X^{\prime} \times Y^{\prime}$ such that $f$ defines a compact operator between them.

Consider first the case $u=0$, treated first in [R1]. Then $f=c$ is a contraction mapping from $Y$ to $Y^{\prime}$. We choose regions $Z$ and $Z^{\prime}$ in $\mathbf{C}^{s}$ that are neighborhoods of $Y$ and $Y^{\prime}$ such that the power series defining $c$ gives a contraction mapping $C: Z \rightarrow Z^{\prime}$ with values in $\operatorname{int}\left(Z^{\prime}\right)$, that is $C(Z) \subset \subset Z^{\prime}$. Let $A=A(Z)$ denote the Banach subalgebra of $\mathscr{C}(Z)$ generated by the coordinate functions $z_{1}, \ldots, z_{s}$, that is the uniform closure of the polynomial functions on $Z$. If the component functions $z_{i} \circ C, i=1, \ldots, s$, are in $A(Z)$ we define $C^{*}: A\left(Z^{\prime}\right) \rightarrow A(Z)$ by $C^{*} a=a \circ C$.

Provided $Z$ is sufficiently small each $z_{i} \circ C$ will lie in $A(Z)$, so $C^{*}$ is defined. Indeed we have

Lemma 2. If $Y$ is a compact subset of $\mathbf{R}^{s}$ and $h$ is holomorphic near $Y$, then on any sufficiently small neighborhood $Z$ of $Y$ in $\mathbf{C}^{s}, h \mid Z$ is a uniform limit of polynomials on $Z$.

Proof. $Y$ is polynomially convex in $\mathbf{C}^{s}$, that is for each $z \in \mathbf{C}^{s}, z \notin Y$ there is a polynomial $p$ with $|p(Y)| \leqq 1,|p(z)|>1$ (for $z \in \mathbf{R}^{s}$ one uses the Weierstrass approximation theorem, for $z_{i} \notin \mathbf{R}$ one uses a polynomial in the $i^{\text {th }}$ variable). 
It follows $([\mathrm{H}], 2.7 .4)$ that every neighborhood of $Y$ contains a polynomial polyhedron (also called a Weil domain) $D$, where for some polynomials $p_{1}, \ldots, p_{n}$ in $z_{1}, \ldots, z_{s}$,

$$
D=\left\{\left(z_{1}, \ldots, z_{s}\right):\left|p_{l}(z)\right| \leqq 1, i=1, \ldots, n\right\}
$$

But $D$ is a Runge domain, i.e. every holomorphic function on $D$ is a uniform limit of polynomials $([\mathrm{H}], 2.7 .7)$, so the lemma follows.

Now choose $Z_{0}$ a compact neighborhood of $c(Z)$ in $\operatorname{int}\left(Z^{\prime}\right)$. Then the restriction operator $A\left(Z^{\prime}\right) \rightarrow A\left(Z_{0}\right)$ is s-compact in the sense of [F2], i.e. it can be approximated by operators of rank $\leqq n^{s}$ with an error whose norm decreases exponentially in $n$. This is shown by truncating power series expansions in $A\left(Z^{\prime}\right)$ after $n$ terms and estimating the error in terms of the gap between $Z_{0}$ and $\operatorname{int}\left(Z^{\prime}\right)$. Since $C^{*}$ factors through this restriction operator, $C^{*}$ is also $s$-compact. This is the result for $u=0$ that we must generalize.

We review some functional analysis that helps to handle functions on a Cartesian product space. Note that for compact regions $W$ in $\mathbf{C}^{u}, Z$ in $\mathbf{C}^{s}$ the image in $A(W \times Z)$ of the tensor product $A(W) \otimes A(Z)$ is dense, since it contains all the coordinate functions. This defines a certain norm on $A(W) \otimes A(Z)$ whose completion is $A(W \times Z)$.

One has, in fact, a norm on any tensor product $B_{1} \otimes B_{2}$ of Banach spaces that generalizes this example and leads to a Banach space completion $B_{1} B_{2}$ of $B_{1} \otimes B_{2}$. In the literature, where various other norms are used, this norm on $B_{1} \otimes B_{2}$ is called the injective norm and $B_{1} B_{2}$ the injective completion (it is variously denoted $B_{1} \hat{\otimes} B_{2}, B_{1} \hat{\otimes}_{\varepsilon} B_{2}$ or $B_{1} \check{\otimes} B_{2}$ ). Quite simply, we regard an element $b_{1} \otimes b_{2}$ as defining a bounded continuous function $\phi\left(b_{1} \otimes b_{2}\right)$ on the product $U_{1} \times U_{2}$ of the unit balls $U_{l}$ in the dual Banach spaces $B_{i}^{*}$, i.e. $\phi\left(b_{1} \otimes b_{2}\right)\left(u_{1}, u_{2}\right)=\left\langle u_{1} \mid b_{1}\right\rangle\left\langle u_{2} \mid b_{2}\right\rangle$. Then $\phi$ extends to a linear embedding $B_{1} \otimes B_{2} \rightarrow \mathscr{C}\left(U_{1} \times U_{2}\right)$ which defines our norm and completion $B_{1} B_{2} \subset \mathscr{C}\left(U_{1} \times U_{2}\right)$.

We have $\mathscr{C}(W) \mathscr{C}(Z)=\mathscr{C}(W \times Z)$, indeed this holds for any pair of compact metric spaces ([Tr], Ex. 44.2). Also if $\alpha_{i}: A_{i} \rightarrow B_{i}$ are bounded linear operators then there is a bounded operator $\alpha_{1} \alpha_{2}: A_{1} A_{2} \rightarrow B_{1} B_{2}$ extending $\alpha_{1} \otimes \alpha_{2}: A_{1} \otimes A_{2} \rightarrow$ $B_{1} \otimes B_{2}$. Such an operator from $A_{1} A_{2}$ to $B_{1} B_{2}$ is called decomposable. If $\alpha_{1}$ and $\alpha_{2}$ are embeddings onto a closed subspace, so is $\alpha_{1} \alpha_{2}$ (hence the name "injective completion"). In particular if $A_{i}$ is a closed subspace of $B_{i}$ then $A_{1} A_{2} \subset B_{1} B_{2}$ is a closed subspace ([Tr], Prop. 43.7 and Cor.). From these facts follow the relation

$$
A(W \times Z)=A(W) A(Z),
$$

which we will use to separate variables.

We note, however, that the evaluation functional $A \otimes A^{*} \rightarrow \mathbf{C}, a \otimes a^{*} \mapsto$ $\left\langle a \mid a^{*}\right\rangle$, does not extend continuously to a map $A A^{*} \rightarrow \mathbf{C}$ for $A$ a Banach space of infinite dimension. For if $k \in B A^{*}$ then the operator $a \mapsto b=\langle k \mid a\rangle, A \rightarrow B$ is a norm limit of finite rank mappings. Indeed $\|k\|$ is the operator norm of $a \mapsto b$ and any operator in the norm closure of finite rank is determined by such a kernel $k$. Thus the evaluation $A \otimes A^{*} \rightarrow \mathbf{C}$ corresponds to the trace on finite rank operators, which is not norm continuous since $\operatorname{dim} A=\infty$.

An operator $\lambda: B \rightarrow A$ is integral if there is a bounded functional $B A^{*} \rightarrow \mathbf{C}$, called the valuation functional relative to $\lambda$, with $b \otimes a^{*} \mapsto\left\langle\lambda(b) \mid a^{*}\right\rangle$. If $\lambda$ is $n$ compact for some $n>0$ then $\lambda$ is integral (using [Tr], Prop. 49.5). 
Now we make our constructions for all $u$. With $X, X^{\prime}$ (respectively $Y, Y^{\prime}$ ) as above we choose compact neighborhoods $W, W^{\prime}$ of $X, X^{\prime}$ in $\mathbf{C}^{u}$ (respectively $Z, Z^{\prime}$ of $Y, Y^{\prime}$ in $\mathbf{C}^{S}$ ) such that $c$ extends to a holomorphic map $C: W^{\prime} \times Z \rightarrow W \times Z^{\prime}$ with values in the interior of $W \times Z^{\prime}$ and such that the components $C_{1}, C_{2}$ of $C$ are uniformly contracting in each variable. Thus $C$ defines a hyperbolic correspondence $F$ from $W \times Z$ to $W^{\prime} \times Z^{\prime}$.

We first define the action $\tilde{C}$ of $C$ on horizontal volume forms

$$
\tilde{C}\left(\phi(w, z) d w_{1} \wedge \cdots \wedge d w_{u}\right)=J(w, z) \phi(C(w, z)) d w_{1} \wedge \cdots \wedge d w_{u},
$$

where $J=\operatorname{det}\left(\frac{\partial}{\partial w^{\prime}}\right) C_{1}$ is the partial Jacobian of $C$ in the horizontal variables. Note that $\tilde{C}$ is the usual pullback of forms followed by the projection that deletes all components containing a $d z_{j}$. We assume that $W^{\prime}$ and $Z$ are chosen so that $w_{l} \circ C$ and $z_{j} \circ C$ belong to $A\left(W^{\prime} \times Z\right)$ for $i=1, \ldots, u$, and $j=1, \ldots, s$. Then we find $\phi \in A\left(W \times Z^{\prime}\right) \Rightarrow J\left(C^{*} \phi\right) \in A\left(W^{\prime} \times Z\right)$. Separating variables, and passing to the space $V(W)$ of volume forms $\phi(w) d w_{1} \wedge \cdots \wedge d w_{u}, \phi \in A(W)$ we obtain a bounded operator

$$
\tilde{C}: V(W) A\left(Z^{\prime}\right) \rightarrow V\left(W^{\prime}\right) A(Z)
$$

As $C\left(W^{\prime} \times Z\right) \subset$ int $\left(W \times Z^{\prime}\right)$, we can factor $\tilde{C}$ through a restriction operator to show it is $(u+s)$-compact.

Our transfer operator $L_{F}$ will be obtained as the partial adjoint of $\tilde{C}$ in the first factor (or in the horizontal variables), that is

$$
L_{F}: V^{*}\left(W^{\prime}\right) A\left(Z^{\prime}\right) \rightarrow V^{*}(W) A(Z)
$$

will satisfy the partial adjoint formula

$$
\left\langle v \mid L_{F}\left(v^{*} \otimes a\right)\right\rangle=\left\langle v^{*} \mid \tilde{C}(v \otimes a)\right\rangle \in A(Z)
$$

for each $v^{*} \in V^{*}\left(W^{\prime}\right), a \in A\left(Z^{\prime}\right)$ and $v \in V(W)$. Clearly this formula defines $L_{F}$ on $V^{*}\left(W^{\prime}\right) \otimes A\left(Z^{\prime}\right)$ and we need only verify continuity. This can be done simply if $\tilde{C}$ can be expanded as an absolutely convergent series $\sum_{i} \xi_{i} \eta_{i}$ of decomposable operators. Then $\left\|\xi_{i} \eta_{l}\right\|=\left\|\xi_{l}\right\|\left\|\eta_{l}\right\|=\left\|\xi_{l}^{*} \eta_{l}\right\|$ so $\sum_{i} \xi_{i}^{*} \eta_{l}$ is bounded, and defines $L_{F}$.

In general we produce $L_{F}$ as follows. We choose compact regions $W_{0}$ and $W_{0}^{\prime}$ in $\mathbf{C}^{u}$ with $W_{0} \subset \subset W$ and $W^{\prime} \subset \subset W_{0}^{\prime}$ such that $C$ analytically continues to $W_{0}^{\prime} \times Z$ with values in int $\left(W_{0} \times Z^{\prime}\right)$. We denote this extension by $C$ as well. The restriction operator $V(W) \rightarrow V\left(W_{0}\right)$ is $u$-compact, hence can be represented by a kernel $k \in V\left(W_{0}\right) V^{*}(W)$. Consider the composite operator $L$

$$
\begin{aligned}
V^{*}\left(W^{\prime}\right) A\left(Z^{\prime}\right) & \rightarrow V^{*}\left(W^{\prime}\right) A\left(Z^{\prime}\right) V\left(W_{0}\right) V^{*}(W) \\
& \rightarrow V^{*}\left(W^{\prime}\right) V\left(W_{0}^{\prime}\right) A(Z) V^{*}(W) \rightarrow V^{*}(W) A(Z)
\end{aligned}
$$

in which (aside from permutations of the factors) the first arrow corresponds to multiplication by $k$, the second arrow corresponds to $I \tilde{C} I$, and the third arrow to the evaluation functional relative to the restriction map $V\left(W_{0}^{\prime}\right) \rightarrow V\left(W^{\prime}\right)$. Then $L$ is clearly continuous and

$$
L\left(v^{*} \otimes a\right)=\sum_{l}\left\langle v^{*} \mid \tilde{C}\left(v_{l} \otimes a\right) \otimes v_{i}^{*}\right\rangle
$$


for $k=\sum v_{l} \otimes v_{i}^{*}, \sum\left\|v_{l}\right\|\left\|v_{i}^{*}\right\|<\infty$. Thus

$$
\left\langle v \mid L\left(v^{*} \otimes a\right)\right\rangle=\left\langle v^{*} \mid \tilde{C}\left(\sum_{i}\left\langle v \mid v_{l}^{*}\right\rangle v_{\imath} \otimes a\right)\right\rangle=\left\langle v^{*} \mid \tilde{C}(v \otimes a)\right\rangle
$$

since $\sum_{i}\left\langle v \mid v_{i}^{*}\right\rangle v_{l}$ is the restriction of $v$ to $W_{0}$. This shows $L=L_{F}$ on $V^{*}\left(W^{\prime}\right) \otimes$ $A\left(Z^{\prime}\right)$, hence that $(*)$ defines $L_{F}$.

We denote $V^{*}(W) A(Z)$ by $K(W, Z)$. As elements of $K(W, Z)$ are kernels (of finite rank operators $V(W) \rightarrow A(Z)$ and their norm limits) we call $L_{F}: K\left(W^{\prime}, Z^{\prime}\right) \rightarrow$ $K(W, Z)$ the kernel transfer of $F$. Choosing $Z_{0}^{\prime} \subset \subset Z^{\prime}$ so that $C$ extends to a map from $W_{0}^{\prime} \times Z$ to $\operatorname{int}\left(W \times Z_{0}^{\prime}\right)$, we can factor $L_{F}$ through a natural operator on kernels

$$
\varepsilon \rho: K\left(W^{\prime}, Z^{\prime}\right) \rightarrow K\left(W_{0}^{\prime}, Z_{0}^{\prime}\right) .
$$

Here $\varepsilon \rho$ decomposes into a $u$-compact extension operator $\varepsilon: V^{*}\left(W^{\prime}\right) \rightarrow V^{*}\left(W_{0}^{\prime}\right)$ and an $s$-compact restriction operator $\rho: A\left(Z^{\prime}\right) \rightarrow A\left(Z_{0}^{\prime}\right)$. Clearly $\varepsilon \rho$ is $(u+s)$-compact, hence $L_{F}$ is also $(u+s)$-compact

Now suppose $r \in A\left(W^{\prime} \times Z\right)$ and consider the operator $r \tilde{C}: V(W) A\left(Z^{\prime}\right) \rightarrow$ $V\left(W^{\prime}\right) A(Z)$,

$$
(r \tilde{C})\left(\phi(w, z) d w_{1} \wedge \cdots \wedge d w_{u}\right)=(J r)(w, z) \phi(C(w, z)) d w_{1} \wedge \cdots \wedge d w_{u} .
$$

Then we can as above form the partial adjoint of $r \tilde{C}$ in the first factor, which we denote

$$
L_{r, F}: K\left(W^{\prime}, Z^{\prime}\right) \rightarrow K(W, Z)
$$

Finally suppose given a positive integer $d$ and a $d \times d$ matrix $r$ over $A\left(W^{\prime} \times Z\right)$. Then we can combine the transfer operators for the entries of $r$ into a block operator on $d$-tuples of kernels that we denote

$$
L_{r, F}: K^{d}\left(W^{\prime}, Z^{\prime}\right) \rightarrow K^{d}(W, Z) .
$$

\section{Section 3. Functoriality of Transfer}

Suppose $f$ (respectively $f^{\prime}$ ) is a hyperbolic correspondence from $X \times Y$ to $X^{\prime} \times Y^{\prime}$ (respectively from $X^{\prime} \times Y^{\prime} \rightarrow X^{\prime \prime} \times Y^{\prime \prime}$ ) for $X, X^{\prime}, X^{\prime \prime}$ regions in $\mathbf{R}^{u}$ and $Y, Y^{\prime}, Y^{\prime \prime}$ regions in $\mathbf{R}^{s}$. Suppose the cross maps $c$ and $c^{\prime}$ are real analytic and that we have complex neighborhoods $W, W^{\prime}, W^{\prime \prime}$ of $X, X^{\prime}, X^{\prime \prime}$ in $\mathbf{C}^{u}$ and $Z, Z^{\prime}, Z^{\prime \prime}$ of $Y, Y^{\prime}, Y^{\prime \prime}$ in $\mathbf{C}^{s}$ such that $c$ and $c^{\prime}$ extend to maps

$$
C: W^{\prime} \times Z \rightarrow W \times Z^{\prime}, \quad C^{\prime}: W^{\prime \prime} \times Z^{\prime} \rightarrow W^{\prime} \times Z^{\prime \prime},
$$

which are uniform limits of polynomials, whose components contract in each variable and with $C^{(l)}\left(W^{(i+1)} \times Z^{(i)}\right) \subset \subset W^{(l)} \times Z^{(l+1)}, i=0,1$. Then as in Sect. 2, the corresponding hyperbolic correspondences $F^{(l)}$ from $W^{(i)} \times Z^{(i)}$ to $W^{(i+1)} \times Z^{(i+1)}$ define operators $L_{F(l)}: K\left(W^{(i+1)}, Z^{(i+1)}\right) \rightarrow K\left(W^{(i)}, Z^{(i)}\right)$. Also $H=F^{\prime} \circ F$ admits a cross map $E: W^{\prime \prime} \times Z \rightarrow W \times Z^{\prime \prime}$ as in Sect. 1, with $E\left(W^{\prime \prime} \times Z\right) \subset \subset W \times Z^{\prime \prime}$. We can then define $L_{H}: K\left(W^{\prime \prime}, Z^{\prime \prime}\right) \rightarrow K(W, Z)$. We will prove

Proposition 2. $L_{H}=L_{F} \circ L_{F^{\prime}}$. 
First we introduce $W_{0}^{(l)} \subset \subset W^{(i)}$ a neighborhood of $X^{(l)}$ such that our cross maps take values in $W_{0}^{(i)} \times Z^{(j)}$, where $(i, j)=(0,1),(1,2)$ and $(0,2)$ for $F, F^{\prime}$ and $H$ respectively. We set $V_{0}^{(i)}=V\left(W_{0}^{(i)}\right)$ and $A^{(i)}=A\left(Z^{(l)}\right)$ and define operators $\tilde{C}, \tilde{C}^{\prime}, \tilde{E}$ from $V_{0}^{(i)} A^{(j)}$ to $V_{0}^{(j)} A^{(i)}$ as in Sect. 2.

The proposition will hold if $L_{F} \circ L_{F^{\prime}}$ is the partial adjoint of $\tilde{E}$ in the first factor. Using formula $(*)$ of Sect. 2, we let $\Sigma_{k} v_{k}^{\prime} \otimes v_{k}^{*} \in V_{0}^{\prime} V^{*}\left(W^{\prime}\right)$ be the kernel of the $u$-compact restriction operator $V\left(W^{\prime}\right) \rightarrow V_{0}^{\prime}$ and we must show that for any $v_{0} \in V_{0}, a^{\prime \prime} \in A^{\prime \prime}$ and $v^{*} \in\left(V_{0}^{\prime \prime}\right)^{*}$,

$$
\left\langle v^{*} \mid \tilde{E}\left(v_{0} \otimes a^{\prime \prime}\right)\right\rangle=\sum_{k}\left\langle v_{k}^{*} \mid \tilde{C}\left(v_{0} \otimes a_{k}^{\prime}\right)\right\rangle
$$

where $a_{k}^{\prime}=\left\langle v^{*} \mid \tilde{C}^{\prime}\left(v_{k}^{\prime} \otimes a^{\prime \prime}\right)\right\rangle$.

We next eliminate $v^{*}$ from this formula. Switching the factors in the domain of $\tilde{C}, \tilde{C}^{\prime}, \tilde{E}$ gives operators

$$
\hat{C}^{(i)}: A^{(i+1)} V_{0}^{(i)} \rightarrow V_{0}^{(l+1)} A^{(l)}, \quad \hat{E}: A^{\prime \prime} V_{0} \rightarrow V_{0}^{\prime \prime} A
$$

Then the proposition reduces to

$$
\hat{E}\left(a^{\prime \prime} \otimes v_{0}\right)=\sum_{k}\left\langle\left(I \hat{C} \circ \hat{C}^{\prime} I\right)\left(a \otimes v_{k}^{\prime} \otimes v_{0}\right) \mid v_{k}^{\prime}\right\rangle
$$

where $I \hat{C}: V_{0}^{\prime \prime} A^{\prime} V_{0} \rightarrow V_{0}^{\prime \prime} V_{0}^{\prime} A$ and $\hat{C}^{\prime} I: A^{\prime \prime} V_{0}^{\prime} V_{0} \rightarrow V_{0}^{\prime \prime} A^{\prime} V_{0}$. Note that $V_{0}^{\prime}$ is the middle factor in the domain and range of $I \hat{C} \circ \hat{C}^{\prime} I$. This version of the proposition can be described as follows:

$\hat{E}$ is the partial trace of $I \hat{C} \circ \hat{C}^{\prime} I$ in the middle factor.

We now calculate this partial trace. We pass from volume forms $\phi d w_{1}$ $\wedge \cdots \wedge d w_{u}$ to functions $\phi$ so as to replace our Banach spaces by Banach algebras of the form $A(R)$. Then with these identifications

$$
\begin{aligned}
\hat{C^{\prime} I}: A\left(W_{0} \times W_{0}^{\prime} \times Z^{\prime \prime}\right) & \rightarrow A\left(W_{0} \times Z^{\prime} \times W_{0}^{\prime \prime}\right), \\
\phi\left(w, w^{\prime}, z^{\prime \prime}\right) & \mapsto \operatorname{det}\left(\frac{\partial}{\partial w^{\prime \prime}} C_{1}^{\prime}\right) \phi\left(w, C^{\prime}\left(w^{\prime \prime}, z^{\prime}\right)\right), \\
I \hat{C}: A\left(W_{0} \times Z^{\prime} \times W_{0}^{\prime \prime}\right) & \rightarrow A\left(Z \times W_{0}^{\prime} \times W_{0}^{\prime \prime}\right), \\
\psi\left(w, z^{\prime}, w^{\prime \prime}\right) & \mapsto \operatorname{det}\left(\frac{\partial}{\partial w^{\prime}} C_{1}\right) \psi\left(C\left(w^{\prime}, z\right), w^{\prime \prime}\right) .
\end{aligned}
$$

Thus $I \hat{C} \circ \hat{C}^{\prime} I=\gamma \mu^{*}$, where $\gamma$ is the product of the 2 Jacobian factors and $\mu: Z \times W_{0}^{\prime} \times W_{0}^{\prime \prime} \rightarrow W_{0} \times W_{0}^{\prime} \times Z^{\prime \prime}$ is the holomorphic map

$$
\left(z, w^{\prime}, w^{\prime \prime}\right) \mapsto\left(C_{1}\left(w^{\prime}, z\right), C_{1}^{\prime}\left(w^{\prime \prime}, C_{2}\left(w^{\prime}, z\right)\right), C_{2}^{\prime}\left(w^{\prime \prime}, C_{2}\left(w^{\prime}, z\right)\right)\right) .
$$


Note that $\mu_{2}\left(z,-, w^{\prime \prime}\right)$ is the composition of $C_{2}(-, z)$ and $C_{1}^{\prime}\left(w^{\prime \prime},-\right)$, hence defines a holomorphic contraction map of $W_{0}^{\prime}$ with values in int $\left(W_{0}^{\prime}\right)$.

We are now in the following general situation. We are given a holomorphic map $v: W \times Z_{1} \rightarrow W \times Z_{2}$ with values in int $(W) \times Z_{2}$, where $W, Z_{1}$ and $Z_{2}$ are regions in complex space and $v$ is uniformly approximable by polynomials. Then for any function $\delta \in A\left(W \times Z_{1}\right)$ the operator

$$
\delta v^{*}: A\left(W \times Z_{2}\right) \rightarrow A\left(W \times Z_{1}\right)
$$

has a partial trace in the factor $W$, which is an operator $\operatorname{Tr}_{W}\left(\delta v^{*}\right): A\left(Z_{2}\right) \rightarrow A\left(Z_{1}\right)$. We are given, moreover, that $v_{1}(-, z)$ is a contraction of $W$ for all $z \in Z_{1}$. The fixed point $p(z)$ of this contraction gives a map $p: Z_{1} \rightarrow W$ that is uniformly approximable by polynomials. Then we have the following formula for the partial trace, in terms of $(p, i d): Z_{1} \rightarrow W \times Z_{1}$ and the components of $v$ :

$$
\operatorname{Tr}_{W}\left(\delta v^{*}\right)=(p, i d)^{*}\left(\frac{\delta v_{2}^{*}}{\operatorname{Det}\left(I-\frac{\partial}{\partial w} v_{1}\right)}\right) .
$$

Note that when $Z_{2}$ is a point, this is just the Atiyah-Bott fixed point formula ([AB], see also [R1, F1]) for a family of holomorphic contraction maps with parameter space $Z_{1}$. In general we can reduce to this case by evaluating both sides on a fixed element of $A\left(Z_{2}\right)$.

Now we apply this partial trace formula to our example. We have $p: W_{0}^{\prime \prime} \times$ $Z \rightarrow W_{0}^{\prime}$ with

$$
C_{1}^{\prime}\left(w^{\prime \prime}, C_{2}\left(w^{\prime}, z\right)\right)=w^{\prime} \text { for } w^{\prime}=p\left(w^{\prime \prime}, z\right) \text {. }
$$

The function $(p, i d)^{*}\left(\gamma / \operatorname{Det}\left(I-\frac{\partial}{\partial w^{\prime}} \mu_{2}\right)\right)$ is just $\operatorname{det}\left(\frac{\partial}{\partial w^{\prime \prime}} E_{1}\right)$, as follows from

Lemma 3. Given $w^{\prime \prime} \in W_{0}^{\prime \prime}$ and $z \in Z$,

$$
\operatorname{det}\left(\left.\frac{\partial E_{1}}{\partial w^{\prime \prime}}\right|_{\left(w^{\prime \prime}, z\right)}\right)=\frac{\operatorname{det}\left(\left.\frac{\partial C_{1}^{\prime}}{\partial w^{\prime \prime}}\right|_{\left(w^{\prime \prime}, z^{\prime}\right)}\right) \operatorname{det}\left(\left.\frac{\partial C_{1}}{\partial w^{\prime}}\right|_{\left(w^{\prime}, z\right)}\right)}{\operatorname{det}\left(I-\left.\left.\frac{\partial C_{1}^{\prime}}{\partial z^{\prime}}\right|_{\left(w^{\prime \prime}, z^{\prime}\right)} \frac{\partial C_{2}}{\partial w^{\prime}}\right|_{\left(w^{\prime}, z\right)}\right)}
$$

where $w^{\prime}=C_{1}^{\prime}\left(w^{\prime \prime}, C_{2}\left(w^{\prime}, z\right)\right)$ and $z^{\prime}=C_{2}\left(C_{1}^{\prime}\left(w^{\prime \prime}, z^{\prime}\right), z\right)$.

Proof. By definition, $E_{1}\left(w^{\prime \prime}, z\right)=C_{1}\left(w^{\prime}, z\right)$. Thus $\frac{\partial}{\partial w^{\prime \prime}} E_{1}=\left(\frac{\partial}{\partial w^{\prime}} C_{1}\right)\left(\frac{\partial}{\partial w^{\prime \prime}} w^{\prime}\right)$. We differentiate $w^{\prime}$ implicitly with respect to $w^{\prime \prime}$ to get

$$
\frac{\partial w^{\prime}}{\partial w^{\prime \prime}}=\frac{\partial C_{1}^{\prime}}{\partial w^{\prime \prime}}+\left(\frac{\partial C_{1}^{\prime}}{\partial z^{\prime}}\right)\left(\frac{\partial C_{2}}{\partial w^{\prime}}\right)\left(\frac{\partial w^{\prime}}{\partial w^{\prime \prime}}\right) .
$$

Solving for $\frac{\partial}{\partial w^{\prime \prime}} w^{\prime}$ gives

$$
\frac{\partial E_{1}}{\partial w^{\prime \prime}}=\frac{\partial C_{1}}{\partial w^{\prime}}\left(I-\frac{\partial C_{1}^{\prime}}{\partial z^{\prime}} \frac{\partial C_{2}}{\partial w^{\prime}}\right)^{-1} \frac{\partial C_{1}^{\prime}}{\partial w^{\prime \prime}},
$$

and the lemma follows. 
Finally we see that $E\left(w^{\prime \prime}, z\right)=\left(\mu_{1}, \mu_{3}\right)\left(z, p\left(w^{\prime \prime}, z\right), w^{\prime \prime}\right)$, that is $E=v_{2} \circ(p, i d)$. Substituting into the partial trace formula gives

$$
\operatorname{Tr}_{W_{0}^{\prime}}\left(I \hat{C} \circ \hat{C}^{\prime} I\right)=\hat{E}
$$

which implies the proposition.

Now we suppose given $r \in A\left(W^{\prime} \times Z\right)$ and $r^{\prime} \in A\left(W^{\prime \prime} \times Z^{\prime}\right)$. We define $t \in$ $A\left(W^{\prime \prime} \times Z\right)$ by

$$
t\left(w^{\prime \prime}, z\right)=r\left(w^{\prime}, z\right) r^{\prime}\left(w^{\prime \prime}, z^{\prime}\right)
$$

where $w^{\prime}$ and $z^{\prime}$ are as in Lemma 2. Then the following result generalizes Proposition 2.

Proposition 3. $L_{t, H}=L_{r, F} \circ L_{r^{\prime}, F^{\prime}}$.

The proof is quite close to that just given, with $\hat{C}, \hat{C^{\prime}}, \hat{E}$ replaced by $r \hat{C}, r^{\prime} \hat{C}^{\prime}, t \hat{E}$ throughout. The function $\gamma$ used above is multiplied by the factor $r\left(w^{\prime}, z\right) r^{\prime}\left(w^{\prime \prime}\right.$, $\left.C_{2}\left(w^{\prime}, z\right)\right)$. If we call this factor $\tau\left(z, w^{\prime}, w^{\prime \prime}\right),(p, i d)^{*} \gamma$ is multiplied by $(p, i d)^{*} \tau=t$, and the proposition follows.

Finally, we note that when $r$ and $r^{\prime}$ are $d \times d$ matrices over $A\left(W^{\prime} \times Z\right)$ and $A\left(W^{\prime \prime} \times Z^{\prime}\right)$ then the formula for $t$ defines an $d \times d$ matrix over $A\left(W^{\prime \prime} \times Z\right)$. The resulting transfer operators on $d$-tuples of kernels still satisfy Proposition 3.

\section{Section 4. The Trace of the Kernel Transfer}

Suppose in Sect. 2 that $W^{\prime}=W$ and $Z^{\prime}=Z$. Then for any square matrix $r$ over $A(W \times Z)$ the kernel transfer $L_{r, F}$ is $(s+u)$-compact and so has a trace. The cross map $C$ contracts $W \times Z$ and so has a unique fixed point $p$. Thus $p$ is also the unique fixed point of $F$.

Proposition 4. $\operatorname{Tr}\left(L_{r, F}\right)=(-1)^{u} \operatorname{Tr}(r(p)) / \operatorname{Det}\left(I-D_{p} F\right)$ when $F$ is a holomorphic map near $p$. Otherwise $\operatorname{Tr}\left(L_{r, F}\right)=0$.

Clearly this reduces to the case $d=1, r \in A(W \times Z)$. Since $L_{r, F}$ is the partial adjoint of $r \tilde{C}$, it follows that $\operatorname{Tr}\left(L_{r, F}\right)=\operatorname{Tr}(r \tilde{C})$. By the fixed point formula of Atiyah-Bott $[\mathrm{AB}]$, cited in Sect. $3, \operatorname{Tr}(r \tilde{C})=J(p) r(p) / \operatorname{Det}\left(I-D_{p} C\right)$, where $J=\frac{\partial}{\partial w^{\prime}} C_{1}$. Thus the proposition reduces to

Lemma 4. $(-1)^{u} \operatorname{Det}\left(I-D_{p} C\right)=J(p) \operatorname{Det}\left(I-D_{p} F\right)$ when $F$ is a holomorphic map near $p$. Otherwise $J(p)=0$.

Proof. From $F\left(C_{1}\left(w^{\prime}, z\right), z\right)=\left(w^{\prime}, C_{2}\left(w^{\prime}, z\right)\right)$, the chain rule gives

$$
\begin{aligned}
D F\left(\begin{array}{cc}
\frac{\partial C_{1}}{\partial w^{\prime}} & \frac{\partial C_{1}}{\partial z} \\
0 & I
\end{array}\right) & =\left(\begin{array}{cc}
I & 0 \\
\frac{\partial C_{2}}{\partial w^{\prime}} & \frac{\partial C_{2}}{\partial z}
\end{array}\right) \text { so }(I-D F)\left(\begin{array}{cc}
\frac{\partial C_{1}}{\partial w^{\prime}} & \frac{\partial C_{1}}{\partial z} \\
0 & I
\end{array}\right) \\
& =\left(\begin{array}{cc}
-I & 0 \\
0 & I
\end{array}\right)(I-D C),
\end{aligned}
$$

and we take determinants to get the desired equation. If $J(p) \neq 0$, then $D_{p} F$ is determined by the above formula so, by the holomorphic inverse function theorem, $F$ is a holomorphic map near $p$. 


\section{Section 5. Zeta Functions for a $C^{\omega}$ System of Hyperbolic Correspondences}

We suppose given integers $u \geqq 0$ and $s \geqq 0$, regions $X_{j}$ in $\mathbf{R}^{u}$ and $Y_{j} \in \mathbf{R}^{s}$, and hyperbolic correspondences $f_{i}$ from $X_{\alpha(l)} \times Y_{\alpha(l)}$ to $X_{\omega(l)} \times Y_{\omega(l)}$ whose cross maps $c_{l}$ are $C^{\omega}$, where the vertices $j$ and the arrows $i$ run over finite index sets $V$ and $A$ and $\alpha, \omega: A \rightarrow K$. We choose $\varepsilon>0$ and let $W_{j}$ (respectively $Z_{j}$ ) be the closed $\varepsilon$-neighborhood of $X_{j}$ (respectively $Y_{j}$ ) in $\mathbf{C}^{u}$ (respectively $\mathbf{C}^{s}$ ). For $\varepsilon$ sufficiently small, $c_{l}$ extends to a holomorphic map $C_{i}: W_{\omega(i)} \times Z_{\alpha(i)} \rightarrow W_{\alpha(i)} \times Z_{\omega(i)}$ with values in int $\left(W_{\alpha(l)} \times Z_{\omega(l)}\right)$ whose components $C_{i 1}, C_{i 2}$ contract uniformly in each factor. Thus $C_{i}$ is the cross map of a hyperbolic correspondence $F_{i}$ from $W_{\alpha(i)} \times Z_{\alpha(i)}$ to $W_{\omega(i)} \times Z_{\omega(l)}$.

In order to iterate this system of correspondences, we suppose that the $c_{l}$ satisfy the hypotheses of Proposition 1 . For $\varepsilon$ sufficiently small, the $C_{i}$ will also satisfy these hypotheses so $F_{i_{n}} \circ \cdots \circ F_{i_{1}}$ is hyperbolic whenever $\omega\left(i_{1}\right)=\alpha\left(i_{2}\right), \ldots, \omega\left(i_{n-1}\right)=$ $\alpha\left(i_{n}\right)$.

Fix $d \geqq 1$. Suppose for each $i$ that we are given a $d \times d$ matrix $r_{i}$ of $C^{\omega}$ functions on $W_{\omega(l)} \times Z_{\alpha(i)}$. For $\varepsilon$ sufficiently small, each $r_{l}$ extends to a $d \times d$ matrix $r_{l} \in M_{d}\left(A\left(W_{\omega(l)} \times Z_{\alpha(l)}\right)\right)$, as shown in Lemma 2 . Then for each $i$ we have the $i^{\text {th }}$ transfer operator

$$
L_{r_{i}, F_{l}}: K^{d}\left(W_{\omega(i)}, Z_{\omega(i)}\right) \rightarrow K^{d}\left(W_{\alpha(i)}, Z_{\alpha(i)}\right) .
$$

We set $K=\oplus_{j} K^{d}\left(W_{j}, Z_{j}\right)$ with the sup norm. Then for each $i$ we let $L_{i}$ be the operator on $K$ which has the block form consisting of the $i^{\text {th }}$ transfer operator in the block at $(\alpha(i), \omega(i))$ with other blocks zero. We define $L=\sum_{i \in A} L_{i}$ to be the kernel transfer for the system of correspondences $F_{i}$ and weights $r_{i}$. Clearly $L$ depends linearly on these weights and $L$ is $n$-compact, $n=u+s$. Accordingly $L$ has a Fredholm determinant, which we can estimate using [F1], Lemma 6:

$$
\log |\operatorname{det}(I-L)| \leqq c\left(1+\log _{+} C\right)^{n+1}
$$

where

$$
L=\sum_{i=1}^{\infty} x_{i} x_{l}^{*},\left|\left\langle x_{l} \mid x_{i}^{*}\right\rangle\right| \leqq C \exp (\beta \sqrt[n]{i}),
$$

and $c$ depends only on $\beta$ and $n$. Factoring our $L$ through a restriction operator, we see that we can take $\beta$ independent of the weights $r_{l}$ and $C$ a constant times the sup norm of these $r_{i}$, so we have shown

Lemma 5. $\log |\operatorname{det}(I-L)| \leqq c\left(1+\log _{+} \sup _{i}\left\|r_{i}\right\|\right)^{u+s+1}$ for some positive constant $c$ independent of the $r_{l}$.

We can calculate this Fredholm determinant det $(I-L)$ provided $\|L\|<1$, using

$$
\operatorname{det}(I-L)=\exp -\sum_{n=1}^{\infty} \frac{1}{n} \operatorname{Tr}\left(L^{n}\right), \quad \operatorname{Tr}\left(L^{n}\right)=\sum_{A^{n}} \operatorname{Tr}\left(L_{i_{1}} \circ \cdots \circ L_{i_{n}}\right) .
$$

We suppose the "arrows" $i_{1}, \ldots, i_{n}$ form an $n$-loop, that is $\omega\left(i_{1}\right)=\alpha\left(i_{2}\right), \ldots$, $\omega\left(i_{n-1}\right)=\alpha\left(i_{n}\right)$ and $\omega\left(i_{n}\right)=\alpha\left(i_{1}\right)$, since the trace term vanishes otherwise. We then use Propositions 1 and 3 to write $L_{i_{1}} \circ \cdots \circ L_{i_{n}}=L_{t, H}$, where $H=H\left(i_{1}, \ldots, i_{n}\right)$ the hyperbolic correspondence $F_{l_{n}} \circ \cdots \circ F_{l_{1}}$ and $t$ is a certain $d \times d$ matrix over $A\left(W_{j}, Z_{j}\right), j=\alpha\left(i_{1}\right)=\omega\left(i_{n}\right)$. Then by Proposition 4,

$$
\operatorname{Tr}\left(L_{i_{1}} \circ \cdots \circ L_{l_{n}}\right)=(-1)^{u} \operatorname{Tr} t(p) / \operatorname{Det}\left(I-D_{p} H\right)
$$


if $H$ is a holomorphic map near its fixed point $p=p\left(i_{1}, \ldots, i_{n}\right)$, and $=0$ otherwise. We make the convention that the determinant is $\infty$ when $H$ is not a holomorphic map near $p$, so this formula continues to hold.

We calculate $t(p)$ as follows. Let $t_{k}=r_{l_{k}}\left(p\left(i_{k}, \ldots, i_{n}, i_{1}, \ldots, i_{k-1}\right)\right)$ for $k=$ $1, \ldots, n$. Then from the definition of $t$ in Proposition 3, we find

$$
t(p)=t_{1} \circ \cdots \circ t_{n}
$$

We let $\Lambda_{n}, n=1,2, \ldots$, consist of the $n$-loops $\left(i_{1}, \ldots, i_{n}\right) \in A^{n}$. We define the multiplicity $\mu$ of such an $n$-loop to be the largest divisor of $n$ such that $i_{k}=i_{\text {f }}$ for $k \equiv \ell\left(\bmod \frac{n}{\mu}\right)$. Thus an $n$-loop of multiplicity $\mu$ is the " $\mu^{\text {th }}$ power" of an $(n / \mu)$ loop of multiplicity 1 . We let $\Lambda_{n}^{\prime} \subset \Lambda_{n}$ be the prime $n$-loops, i.e. the $n$-loops of multiplicity 1 .

Now we consider a prime $n$-loop $\vec{i}=\left(i_{1}, \ldots, i_{n}\right)$ and its cyclic permutations and their powers and assess their contribution $Z_{\vec{i}}$ to det $(I-L)$. We obtain

$$
\operatorname{det}(I-L)=\prod_{n=1}^{\infty} \prod_{\vec{i} \in \Lambda_{n}^{\prime \prime}} Z_{\vec{i}}
$$

where $\Lambda_{n}^{\prime \prime} \subset \Lambda_{n}^{\prime}$ and every prime $n$-loop is a cyclic permutation of just one element of $\Lambda_{n}^{\prime \prime}$. Then

$$
Z_{\vec{i}}=\exp -\sum_{m=1}^{\infty} \frac{(-1)^{u}}{m} \operatorname{Tr}(t(p))^{m} / \operatorname{Det}\left(I-\left(D_{p} H\right)^{m}\right)
$$

with $p$ and $t(p)=t_{1} \circ \cdots \circ t_{n}$ as above. In deriving this expression we use that $\operatorname{Tr}\left(t_{k} \circ \cdots \circ t_{n} \circ t_{1} \circ \cdots \circ t_{k-1}\right)=\operatorname{Tr}(t(p))^{m}$ for all $k$, and a similar observation for the determinantal factor. The cyclic permutations of the $m^{\text {th }}$ power of $\vec{i}$ contribute then $n$ equal terms, and the factor $\frac{1}{m}$ arises as $n\left(\frac{1}{n m}\right)$. We summarize this as

Proposition 5. For weights $r_{i}$ with $\|L\|<1$,

$$
\operatorname{det}(I-L)=\prod_{n=1}^{\infty} \prod_{\vec{i} \in \Lambda_{n}^{\prime \prime}} \exp -\sum_{m=1}^{\infty} \frac{(-1)^{u}}{m} \frac{\operatorname{Tr}(t(p))^{m}}{\operatorname{Det}\left(I-\left(D_{p} H\right)^{m}\right)}
$$

Observe that the product on the right is absolutely convergent for $\|L\|<1$. Note that the $r_{l}$ 's only enter linearly into $L$, so the left-hand side is an entire function on this space of weights. So we have analytically continued this product to all choices of weights $\left(r_{i}\right) \in \oplus_{i \in A} M_{d}\left(A\left(W_{\omega(i)} \times Z_{\alpha(i)}\right)\right)$. Note also that the terms on the right are independent of our choice of complex neighborhoods.

\section{Section 6. Zeta Functions for a Hyperbolic Set of a Flow}

Suppose that $M$ is a manifold with a $C^{1}$ flow $\phi_{t}: M \rightarrow M$, and $\Omega \subset M$ is a compact invariant set. If $\phi$ has no stationary points in $\Omega$, there is a line subbundle $E^{c} \subset$ $T_{\Omega} M$ invariant by the lifted flow $D \phi_{t}: T_{\Omega} M \rightarrow T_{\Omega} M$, such that a section of $E^{c}$ is a multiple of the vectorfield that generates $\phi$ on $\Omega$. We say $\Omega$ is a hyperbolic set for 
$\phi$ if $T_{\Omega} M / E^{c}$ is the direct sum of 2 continuous invariant subbundles, one contracted by $D \phi_{t}$ for $t \gg 0$ and the other contracted by $D \phi_{t}$ for $t \ll 0$. Then we find a continuous invariant splitting $T_{\Omega} M=E^{u} \oplus E^{s} \oplus E^{c}$, where $E^{s}$ is contracted by $D \phi_{t}$ for $t \gg 0$ and $E^{u}$ is contracted by $D \phi_{t}$ for $t \ll 0$. Given $\delta>0$, the metric on $T_{\Omega} M$ can be chosen (take any metric and average it over a long time interval $[-\tau, \tau])$ so we have uniform contraction for $t \geqq \delta$ or $t \leqq-\delta$, respectively. We set $u=\operatorname{dim} E^{u}$ and $s=\operatorname{dim} E^{s}$, so $u+s+1=\operatorname{dim}(M)$. One calls $E^{c}, E^{u}$ and $E^{s}$ the center, unstable and stable bundles in $T_{\Omega} M$.

Suppose that we are given a (real or complex) vector bundle $\xi$ over $\Omega$ with projection $\pi: \xi \rightarrow \Omega$. A continuous flow $\psi_{t}: \xi \rightarrow \xi$ with $\pi \circ \psi_{t}=\phi_{t} \circ \pi$ such that $\psi_{t}: \pi^{-1}(q) \rightarrow \pi^{-1}\left(\phi_{t} q\right)$ is a linear map for all $q \in \Omega$ and $t \in \mathbf{R}$ is called a lift of $\phi$. We call a pair of lifts $\left(\psi^{+}, \psi^{-}\right)$, relative to a pair of vector bundles $\left(\xi^{+}, \xi^{-}\right)$ over $\Omega$, a virtual lift $\psi^{ \pm}$of $\phi$ to the virtual bundle $\xi^{ \pm}$. Setting $\xi^{-}=0, \xi^{+}=\xi$ and $\psi^{+}=\psi$ we identify any lift $\psi$ with a corresponding virtual lift $(\psi, 0)$.

Now we consider any periodic orbit $\gamma$ of $\phi$ in $\Omega$, determined by a point $q \in \Omega$ and a positive number $\ell$ with $\phi_{\ell}(q)=q$. Of course $(q, \ell)$ and $\left(\phi_{t}(q), \ell\right)$ determine the same $\gamma$, for all $t \in \mathbf{R}$. If the smallest $t>0$ with $\phi_{t}(q)=q$ is $\ell / \mu$ we say $\gamma$ has period $\ell=\ell(\gamma)$ and multiplicity $\mu=\mu(\gamma)$. If $\mu(\gamma)=1$ we say $\gamma$ is prime.

Given a lift $\psi$ of $\phi \mid \Omega$ we define the holonomy of $\psi$ over $\gamma$ to be the similarity class of the transformation $\psi_{\ell(\gamma)}: \xi_{q} \rightarrow \xi_{q}$, and the $\gamma$-character $\chi_{\gamma}(\psi)$ to be the trace of this transformation. Clearly this trace does not change when $q$ is replaced by $\phi_{t} q, t \in \mathbf{R}$, so $\chi_{\gamma}$ depends only on $\gamma$. Given a virtual lift $\psi^{ \pm}$, we define $\chi_{\gamma}\left(\psi^{ \pm}\right)=$ $\chi_{\gamma}\left(\psi^{+}\right)-\chi_{\gamma}\left(\psi^{-}\right)$. The linear Poincaré map $\mathbf{P}_{\gamma}$ is the holonomy of the natural lift of $\phi$ to $T_{\Omega} M / E^{c} \cong E^{u} \oplus E^{s}$. Clearly $\mathbf{P}_{\gamma}=U_{\gamma} \oplus S_{\gamma}$, where $U_{\gamma}$ is a linear expansion of $E^{u}$ and $S_{\gamma}$ is a linear contraction of $E^{s}$.

Consider the series

$$
\sum_{\gamma} \frac{1}{\mu(\gamma)} \frac{\chi_{\gamma}\left(\psi^{ \pm}\right)}{\left|\operatorname{Det}\left(I-\mathbf{P}_{\gamma}\right)\right|},
$$

where $\gamma$ runs over all periodic orbits of $\phi \mid \Omega$. Provided $\psi_{t}^{ \pm}$are sufficiently contractive as $t \rightarrow+\infty$, this series is absolutely convergent for the following reason. The number $N(t)$ of $\gamma$ with $\ell(\gamma) \leqq t$ satisfies $\lim \sup _{t \rightarrow \infty} \frac{1}{t} \log N(t) \leqq h\left(\phi_{1} \mid \Omega\right)<\infty$, where $h$ denotes topological entropy. The growth rate of a lift $\psi$ is $\operatorname{gr}(\psi)=$ $\lim _{t \rightarrow \infty} \sup \frac{1}{t} \log \left\|\psi_{t}\right\|<\infty$, where the choice of fiber metric on $\xi$ is irrelevant since $\Omega$ is compact. To estimate the denominator, we write

$$
\left|\operatorname{Det}\left(I-\mathbf{P}_{\gamma}\right)\right|=\left|\operatorname{Det}\left(I-S_{\gamma}\right)\right|\left|\operatorname{Det}\left(I-U_{\gamma}\right)\right|=\left|\operatorname{Det}\left(U_{\gamma}\right)\right|(1+o(1))
$$

and note that $\lim _{\gamma} \inf \frac{1}{\ell(\gamma)} \log \left|\operatorname{det} U_{\gamma}\right| \geqq|g|$, where $g \leqq 0$ is the growth rate of the natural lift $\Lambda^{u}\left(D \phi_{-t} \mid E^{u}\right)$ of the time reversed flow $\phi_{-t}$ to the line bundle $\Lambda^{u}\left(E^{u}\right)$. If $\operatorname{gr}(\psi)<|g|-h\left(\phi_{1} \mid \Omega\right)$ then, breaking the sum over $\gamma$ up into a sum over $n \in \mathbf{Z}$ and over those $\gamma$ with $e^{n-1} \leqq \ell(\gamma)<e^{n}$, we see this series is absolutely convergent.

For $\psi^{ \pm}$with gr $\left(\psi^{ \pm}\right)<|g|-h\left(\phi_{1} \mid \Omega\right)$ we define

$$
\zeta\left(\psi^{ \pm}\right)=\exp -\sum_{\gamma} \frac{1}{\mu(\gamma)} \frac{\chi_{\gamma}\left(\psi^{ \pm}\right)}{\left|\operatorname{Det}\left(I-\mathbf{P}_{\gamma}\right)\right|} .
$$

We regard $\Omega, \phi$, and $\xi^{ \pm}$as fixed but the virtual lift $\psi^{ \pm}$as a parameter. In particular, for $\xi$ a complex vector bundle any lift $\psi$ of $\phi$ to $\xi$ determines a family $\psi^{z}$ 
of lifts involving one complex parameter $z$ with $\psi_{t}^{z}=e^{-t z} \psi_{t}$. As $\operatorname{gr}\left(\psi^{z}\right) \leqq \operatorname{gr}(\psi)-$ $\operatorname{Re}(z), \psi^{z}$ lies in the domain of $\zeta$ if $\operatorname{Re} z$ is sufficiently large. We define the flattrace function

$$
\begin{aligned}
T^{b}(z) & =\zeta\left(\psi^{ \pm z}\right) \\
& =\exp -\sum_{\gamma} \frac{1}{\mu(\gamma)} \frac{\chi_{\gamma}\left(\psi^{ \pm}\right)}{\left|\operatorname{Det}\left(I-\mathbf{P}_{\gamma}\right)\right|} e^{-z \ell(\gamma)},
\end{aligned}
$$

where on any compact subset of the halfplane

$$
\operatorname{Re} z>\operatorname{gr}\left(\psi^{ \pm}\right)+h\left(\phi_{1} \mid \Omega\right)+\operatorname{gr}\left(\Lambda^{u}\left(D \phi_{-t} \mid E^{u}\right)\right)
$$

the series in $\gamma$ is uniformly convergent. Thus $T^{b}$ is holomorphic on this halfplane. Clearly $T^{b}(z)=T_{+}^{b}(z) / T_{-}^{b}(z)$, where $T_{+}^{b}(z)$ and $T_{-}^{b}(z)$ are defined using $\chi_{\gamma}\left(\psi^{+}\right)$and $\chi_{\gamma}\left(\psi^{-}\right)$in place of $\chi_{\gamma}\left(\psi^{ \pm}\right)$. If necessary, we write $T_{\psi}^{b}(z)$ or $T_{\psi^{ \pm}}^{b}(z)$ for $T^{b}(z)$.

We define the flat-trace of the operator $\psi_{t}^{*}$ of $\psi_{t}$ on continuous sections of $\xi$ to be the following atomic measure on $(0, \infty)$ concentrated on the lengths of periodic orbits

$$
\operatorname{Tr}^{b} \psi_{t}^{*}=\sum_{\gamma} \frac{\ell(\gamma)}{\mu(\gamma)} \frac{\chi_{\gamma}(\psi)}{\left|\operatorname{Det}\left(I-\mathbf{P}_{\gamma}\right)\right|} \delta(x-\ell(\gamma))
$$

(cf. [Gu, GS]). To understand the sense in which this measure is a "trace", at least for $C^{\infty}$ flows and lifts, see [GS], Chapter 6. In brief, even though the averaged operators $\int_{0}^{\infty} \alpha(t) \psi_{t}^{*} d t$ for $\alpha(t)$ continuous with compact support do not have continuous kernels, one may introduce a many-parameter deformation of $\phi_{t}, \psi_{t}$ to obtain averaged operators with continuous kernels. Their traces define a measure on the parameter space which restricts to the flat-trace.

The relation of $T_{\psi}^{b}(z)$ to the flat-trace of $\psi_{t}^{*}$ is clear (cf. [F3], Sect. 5):

$$
T_{\psi}^{b}(z)=\exp -\mathscr{L}\left(\frac{1}{t} \operatorname{Tr}^{b} \psi_{t}^{*}\right)(z),
$$

where $\mathscr{L}(v): z \mapsto \int_{0}^{\infty} e^{-t z} d v(t)$ denotes the Laplace transform of a measure $v$ on the ray $t>0$. In some formal sense, $T_{\psi}^{b}(z)$ is a Fredholm determinant for the infinitesimal generator $X_{\psi}$ of $\psi_{t}^{*}$. Indeed consider the definition for the zeta-regularized determinant of an elliptic operator $\Delta$ with positive symbol and no zero eigenvalues on a closed manifold:

$$
\operatorname{det}_{\zeta} \Delta=\exp -\left.\frac{d}{d s}\right|_{s=0} \operatorname{Tr} \Delta^{-s}
$$

where

$$
\Delta^{-s}=\frac{1}{\Gamma(s)} \int_{0}^{\infty} t^{s-1} e^{-t \Delta} d t, \quad \operatorname{Re} s \ll 0,
$$

and where $\operatorname{Tr} \Delta^{-s}$ is analytically continued to $s=0$. If the underlying manifold has odd dimension then $\left.\operatorname{Tr} \Delta^{-s}\right|_{s=0}=0$, so

$$
\operatorname{det}_{\zeta} \Delta=\exp -\left.\int_{0}^{\infty} t^{s-1} \operatorname{Tr} e^{-t \Delta} d t\right|_{s=0} .
$$


Replacing the generator $-\Delta$ of $e^{-t \Delta}$ by the generator $X_{\psi}-z$ of $\left(\psi_{t}^{z}\right)^{*}$, and trace by flat-trace, we make the definition

$$
\operatorname{det}_{\zeta}\left(z-X_{\psi}\right)=T_{\psi}^{b}(z) .
$$

More precisely, the generator of $\left(\psi_{t}^{z}\right)^{*}$ is $X_{\psi}-z E$, where $E$ denotes the Euler vector field on $\xi$ which is tangent to the fibers and generates the dilation flow $v \mapsto e^{t} v$ on $\xi$, so one should write here $\operatorname{det}_{\zeta}\left(z E-X_{\psi}\right)$.

For a virtual lift, we let $\operatorname{Tr}^{b} \psi_{t}^{ \pm, *}=\operatorname{Tr}^{b} \psi_{t}^{+, *}-\operatorname{Tr}^{b} \psi_{t}^{-, *}$, so $T^{b}(z)=$ $\exp -\mathscr{L}\left(t^{-1} \operatorname{Tr}^{b} \psi_{t}^{ \pm, *}\right)$. Most of the dynamical zeta functions in one variable that one associates to $\phi_{t}$ and its hyperbolic set $\Omega$ are of the form $T^{b}(z)$ for some choice of $\xi^{ \pm}$and $\psi_{t}^{ \pm}$. Consider, for example, the Ruelle function and the Selberg function

$$
R(z)=\prod_{\gamma: \mu(\gamma)=1}\left(1-e^{-z \ell(\gamma)}\right), \quad S(z)=\exp -\sum_{\gamma} \frac{1}{\mu(\gamma)} \frac{e^{-z /(\gamma)}}{\operatorname{Det}\left(I-S_{\gamma}\right)},
$$

where $\gamma$ runs over the periodic orbits of $\phi_{t} \mid \Omega$. To write $S(z)=T^{b}(z)$ we find $\xi^{ \pm}, \psi^{ \pm}$ so that for any $\gamma$

$$
\left|\operatorname{Det}\left(I-\mathbf{P}_{\gamma}\right)\right|=\operatorname{Det}\left(I-S_{\gamma}\right) \chi_{\gamma}\left(\psi^{ \pm}\right) .
$$

Let $\varepsilon_{\gamma}=\operatorname{sgn} \operatorname{det}\left(I-\mathbf{P}_{\gamma}\right)$. Then this equation reduces to

$$
\chi_{\gamma}\left(\psi^{ \pm}\right)=\varepsilon_{\gamma} \operatorname{det}\left(I-U_{\gamma}\right)=\varepsilon_{\gamma} \sum_{j=0}^{\infty}(-1)^{j} \operatorname{Tr}\left(\Lambda^{j} U_{\gamma}\right)
$$

The natural lift $\Lambda^{j}\left(D \phi_{t} \mid E^{u}\right)$ of $\phi_{t} \mid \Omega$ to $\Lambda^{j} E^{u}$ has holonomy $\operatorname{Tr}\left(\Lambda^{j} U_{\gamma}\right)$. On the other hand $\varepsilon_{\gamma}=\operatorname{sgn} \operatorname{det}\left(-U_{\gamma}\right)$, since $\left(I-U_{\gamma}\right)=-U_{\gamma}\left(I-U_{\gamma}^{-1}\right)$ and $\operatorname{det}\left(I-U_{\gamma}^{-1}\right)>0$. Thus $(-1)^{u} \varepsilon_{\gamma}=s g n \operatorname{det} U_{\gamma}$ is the holonomy of the flat unstable orientation line bundle $w$ of $E^{u}$ for its flat lift. We choose

$$
\xi^{ \pm}=\left(\Lambda^{ \pm(-1)^{u}} E^{u}\right) \otimes w,
$$

where $\Lambda^{+} \xi$ and $\Lambda^{-} \xi$ denote the direct sum of the exterior powers $\Lambda^{j} \xi$ over $j$ even and $j$ odd. If $\psi^{ \pm}$is the tensor product of the lifts on each factor then $\chi_{\gamma}\left(\psi^{ \pm}\right)$has the desired form, so $S(z)=T_{\psi^{ \pm}}^{b}(z)$.

Next we note $-\log R(z)=\sum_{\gamma} \frac{1}{\mu(\gamma)} e^{-z /(\gamma)}$. To arrange $R(z)=T^{b}(z)$ we find $\xi^{ \pm}, \psi^{ \pm}$so that for any $\gamma$

$$
\left|\operatorname{Det}\left(I-\mathbf{P}_{\gamma}\right)\right|=\chi_{\gamma}\left(\psi^{ \pm}\right)
$$

We find that

$$
\xi^{ \pm}=\left(\Lambda^{ \pm(-1)^{u}}\left(T M / E^{c}\right)\right) \otimes w
$$

with the natural lifts $\psi^{ \pm}$does the trick.

If we are given a lift $\rho_{t}$ of $\phi_{t}$ to a bundle $\eta$, we define the corresponding Ruelle and Selberg functions

$$
\begin{aligned}
& R_{\rho}(z)=\exp -\sum_{\gamma} \frac{1}{\mu(\gamma)} e^{-z \ell(\gamma)} \chi_{\gamma}(\rho) \\
& S_{\rho}(z)=\exp -\sum_{\gamma} \frac{1}{\mu(\gamma)} e^{-z /(\gamma)} \frac{\chi_{\gamma}(\rho)}{\operatorname{det}\left(I-S_{\gamma}\right)} .
\end{aligned}
$$


To choose $\xi^{ \pm}$and $\psi^{ \pm}$for which $R_{\rho}(z)=T^{b}(z)$ or $S_{\rho}(z)=T^{b}(z)$, we tensor the bundles and lifts used for $R(z)$ or $S(z)$ with $\eta$ and $\rho$. In particular, we are interested in $R_{\rho}(z)$ for $\eta$ a flat bundle of degree $d$ on $M$ with flat lift $\rho$ and holonomy given by $\alpha: \pi_{1} M \rightarrow G l(d, \mathbf{C})$. Then

$$
R_{\rho}(z)=\prod_{\gamma: \mu(\gamma)=1} \operatorname{det}\left(I-e^{-z /(\gamma)} \alpha(\gamma)\right) .
$$

The torsion function of such a representation $\alpha$ is

$$
Z_{\alpha}(z)=\exp -\sum_{\gamma} \frac{1}{\mu(\gamma)} e^{-z /(\gamma)} \varepsilon_{\gamma} \operatorname{Tr}(\alpha(\gamma)) .
$$

Here we choose simply $\xi^{ \pm}=\eta \otimes \Lambda^{ \pm}\left(T M / E^{c}\right)$, with the natural lifts $\psi^{ \pm}$(flat on the $\eta$ factor and induced by $D \phi_{t}$ on the other). These are the dynamical zeta functions that carry topological information about the flow. It is so named because of the cases where the value of $Z_{\alpha}(z)$ at $z=0$ can be defined by analytic continuation and identified with the Reidemeister torsion $\tau_{\alpha}\left(N, N_{-}\right)$for a certain pair of spaces $\left(N, N_{-}\right)$(here $\Omega$ is isolated, $N$ an isolating block and $N_{-}$its exit set, see $[\mathrm{F} 3, \mathrm{~F} 4, \mathrm{MS}, \mathrm{S}])$.

The relationship between Ruelle and Selberg functions was worked out in [F1], Proposition 2:

$$
R_{\rho}(z)=S_{\rho^{+}}(z) / S_{\rho^{-}}(z),
$$

where $\rho^{ \pm}=\rho \otimes \Lambda^{ \pm}\left(D \phi_{t} \mid E^{s}\right)$. The above expansions of $R_{\rho}$ and $S_{\rho}$ as $T_{\psi^{+}}^{b}(z) / T_{\psi^{-}}^{b}(z)$ are quite analogous. We tend to regard the flat-trace functions $T_{\psi}^{b}(z)$ as building blocks and functions such as $R(z)$ and $Z_{\alpha}(z)$ as the zeta functions of primary interest. However, even the case $\xi=\Omega \times \mathbf{C}, \psi_{t}$ trivial, leads to the correlation zeta function

$$
d(z)=\exp -\sum_{\gamma} \frac{1}{\mu(\gamma)} \frac{1}{\left|\operatorname{Det}\left(I-\mathbf{P}_{\gamma}\right)\right|} e^{-z /(\gamma)}
$$

which is of special interest in statistical physics [Ru].

The class of zeta functions $T^{b}(z)$ can be widened when one is given a continuous weight function $a$ on $\Omega$ with $\operatorname{Re}(a)>0$. Then a given lift $\psi$ can be deformed to $\psi^{z a}$ with

$$
\psi^{z a}(v)=\exp \left(-z \int_{0}^{t} a\left(\phi_{s} p\right) d s\right) \psi v,
$$

where $p=\pi(v), v \in \xi$. One has

$$
\chi_{\gamma}\left(\psi^{a}\right)=\exp \left(-\int_{0}^{\ell(\gamma)} a\left(\phi_{s} p\right) d s\right) \chi_{\gamma^{\prime}}(\psi),
$$

where $\gamma$ passes through $p$.

When given a virtual lift $\psi^{ \pm}$we set

$$
\zeta\left(\psi^{ \pm, z a}\right)=\zeta\left(\psi^{+, z a}\right) / \zeta\left(\psi^{-, z a}\right) .
$$

Of course for $a=1$ we recover the function $T^{b}(z)$. For $a=\log \left|J_{u}\right|$, where $J_{u}$ is the unstable expansion of $\phi_{t}$ relative to some fiber metric on $T_{\Omega} M$, we obtain the differential zeta function of Parry [Pa]. 
While the zeta functions we know of in the literature are all of this form $\zeta\left(\psi^{ \pm, z a}\right)$, one can define a more general class, as follows. A zeta function of one variable for $(\Omega, \phi)$ is the ratio $\zeta\left(\psi^{+}(z)\right) / \zeta\left(\psi^{-}(z)\right)$, where $\psi^{+}(z)$ and $\psi^{-}(z)$ are lifts of $\phi \mid \Omega$ depending holomorphically on a parameter $z \in \mathbf{C}$ such that $\left\|\psi_{1}^{+}(x)\right\|$, $\left\|\psi_{1}^{-}(x)\right\| \rightarrow 0$ as $x \rightarrow+\infty, x \in \mathbf{R}$. Then this zeta function is holomorphic on some ray $[c, \infty)$.

\section{Section 7. Meromorphic Extension of $T^{b}(z)$ and $\zeta(\psi)$}

There are two sorts of obstructions to extending $T^{b}(z)$ to a meromorphic function on $\mathbf{C}$.

The first is topological. Suppose $\phi$ is the suspension flow of the Smale horseshoe $f: S^{2} \rightarrow S^{2}$ with return time 1 . Let $\Omega$ be an invariant set in the suspended invarient cantor set so $u=s=1$. Then the corresponding Ruelle function

$$
R(z)=\left.\prod_{\gamma: \mu(\gamma)=1}\left(1-x^{\prime(\gamma)}\right)\right|_{x=e^{-z}}=\zeta_{\Omega}\left(e^{-z}\right)^{-1},
$$

where the power series $\zeta_{\Omega}(x)$ is the Artin-Mazur generating function for $f \mid\left(\Omega \cap S^{2}\right)$. It is possible to find invariant sets in the horseshoe whose Artin-Mazur function is a rather general subproduct of $\zeta_{\Omega}(x)$, hence does not extend meromorphically beyond some radius (e.g. $|x|=1$ in $[\mathrm{BL}]$, p. 47, as follows from the Fabry gap theorem, cf. [B]II, p. 296).

We avoid this difficulty by restricting to the case when $\Omega$ is isolated, i.e. $\Omega=$ $\bigcap_{-\infty}^{+\infty} \phi_{t}(U)$ for some open set $U \subset M$. An isolated hyperbolic set if a basic set in Smale's terminology.

For $\Omega$ basic, there is still an obstruction - the smoothness of the flow. If $\phi$ is not $C^{r}$ for some $r<\infty$ then there is sometimes no meromorphic extension to $\mathbf{C}$ $[\mathrm{Ga}, \mathrm{P}, \mathrm{PP}]$. Examples can be constructed on suspension flows of horseshoes with variable return time. Accordingly we must assume $\psi, \phi$ are $C^{\infty}$. By analogy with the well-understood case of expanding maps, this should be enough ([T, R2, F5]) but the case when they are $C^{\omega}$ should be simpler ([R1, F1]).

We now fix a $C^{\omega}$ flow $\phi$ and a compact basic set $\Omega$ for $\phi$. We assume also that $\left(\xi^{ \pm}, \psi^{ \pm}\right)$is a $C^{\omega}$ virtual lift, with $\xi^{ \pm}$complex vector bundles over $\Omega$.

Theorem. $T^{b}(z)$ extends to a meromorphic function on $\mathbf{C}$ of order at most $\operatorname{dim}(M)$. Moreover $\zeta\left(\psi^{+}\right) / \zeta\left(\psi^{-}\right)$is a meromorphic function of $\psi_{t}^{ \pm}$and every zeta function of one variable for $(\Omega, \phi)$ extends meromorphically to $\mathbf{C}$.

Referring to the examples of Sect. 6 , we see that for $\xi, \psi$ of class $C^{\omega}$, the Ruelle function $R_{\psi}(z)$ extends to a meromorphic function on $\mathbf{C}$. (On the other hand, we cannot continue the Selberg function $S_{\psi}(z)$, or even $S(z)$, using this theorem unless the stable bundle is $C^{\omega}$.) We apply this to the case when $M=U Q$ is the unit tangent bundle of a closed manifold $Q$ with a $C^{\omega}$ Riemannian metric of negative curvature, $\phi$ is the geodesic flow on $M$ and $\Omega=M$. In this case we obtain a result in which no flow is mentioned.

Corollary. For a closed Riemannian manifold $Q$ of negative curvature, the product

$$
R(z)=\prod_{\gamma} 1-e^{-z f(\gamma)}, \quad \operatorname{Re} z \gg 0
$$


where $\gamma$ ranges over all prime closed oriented geodesics on $Q$, extends to a meromorphic function on $\mathbf{C}$. Similarly, the Ruelle and torsion functions of a matrix representation $\alpha: \pi_{1}(U Q) \rightarrow G l(d ; \mathbf{C})$

$$
R_{\alpha}(z)=\prod_{\gamma} \operatorname{det}\left(I-e^{-z /(\gamma)} \alpha(\gamma)\right)
$$

and

$$
Z_{\alpha}(z)=\prod_{\gamma} \operatorname{det}\left(I-\sigma_{\gamma} e^{-z f(\gamma)} \alpha(\gamma)\right)^{\varepsilon}, \quad \varepsilon=(-1)^{\operatorname{dim} Q-1}
$$

have meromorphic extensions to $\mathbf{C}$. Here $\sigma_{\gamma}=1$ or -1 as $\gamma$ preserves or reverses the orientation of $Q$. These functions have order at most $2(\operatorname{dim} Q)-1$.

This corollary opens up the possibility of exploring the special values of these functions, which were previously only known to exist for $Q$ locally symmetric. In particular, if there are no $\alpha$-twisted harmonic forms on $U Q$ (i.e. no nonzero $d$-tuple of harmonic forms on the universal cover of $U Q$ is $\alpha$-equivariant) we conjecture

$$
Z_{\alpha}(0)=\tau_{\alpha}(U Q)
$$

where $\tau_{\alpha}$ is the Reidemeister torsion of the manifold $U Q$. This is a sort of "Lefschetz formula" relating the periodic orbits of the geodesic flow over $Q$ to the topology of $Q$ [F3]. It is known when $Q$ is R-hyperbolic, i.e. of constant negative curvature, and $\alpha$ unitary ([F6, F7] ). Note that our condition on harmonic forms implies that $\alpha$ is acyclic but the converse only holds for unitary $\alpha$-see [F4] for some pathological situations where this distinction seems to matter.

In other examples, the special value of a ratio of Selberg functions is of geometric interest. For $Q$ hyperbolic with $\operatorname{dim} Q=2 k+1$, Millson expressed $e^{\pi i \eta}, \eta$ the eta invariant of $Q$, as the value $S_{\rho^{+}}(z) /\left.S_{\rho^{-}}(z)\right|_{z=k}$, where $\rho^{ \pm}$are the natural lifts of the geodesic flow on $U Q$ to the spin bundles $\xi^{ \pm}$with $\xi_{v}^{+} \oplus \xi_{v}^{-}=\Lambda^{k}\left(T_{p} Q / \mathbf{R}_{v}\right)$ for $v \in U Q, p=\pi(v)[\mathrm{M}]$. For $Q \mathbf{C}$-hyperbolic (i.e. Kähler with constant negative holomorphic sectional curvature) the holomorphic torsion invariants of Ray-Singer are expressible as such special values of Selberg functions [F8]. One may hope to generalize these results to variable negative curvature using our corollary.

Proof of the theorem. Clearly we may reduce to the case of a $C^{\omega}$ lift $(\xi, \psi)$. For some small neighborhood $U$ of $\Omega, \xi$ extends to a $C^{\omega}$ bundle on $U$ and $\psi$ to a $C^{(\omega)}$ family of bundle maps $\left.\left.\xi\right|_{U \cap \phi_{-t} U} \rightarrow \xi\right|_{U \cap \phi_{t} U}$, which we will also denote $(\xi, \psi)$.

Since $\phi$ has no stationary points in $\Omega$ and $\Omega$ is compact, we can choose a finite number of small transverse compact disjoint $C^{(\omega)}$ discs $D_{k}$ of codimension 1, such that for every $p \in \Omega$ there is a $t \in(0,1)$ with $\phi_{t} p \in \bigcup_{k} \operatorname{int}\left(D_{k}\right)$. We choose a basis of $C^{(1)}$ sections for $\xi \mid D_{k}$, so $\xi \mid D_{k} \cong D_{k} \times \mathbf{C}^{d}, d=\operatorname{rank}(\xi)$.

Now we fix $\delta>0$ so that if $p, \phi_{t} p \in \bigcup_{k} D_{k}$ and $t>0$ then $t>\delta$. We choose a $C^{(1)}$ Riemannian metric on $U$ and a constant $\sigma<1$ so that

$$
\left\|D \phi_{\delta}\left|E^{s}(\Omega)\|<\sigma, \quad\| D \phi_{-\delta}\right| E^{u}(\Omega)\right\|<\sigma .
$$

Consider a point $p \in \Omega \cap \operatorname{int}\left(D_{k}\right)$ and the $C^{\omega}$ coordinates $\left(x_{1}, \ldots, x_{u+s}\right),\|x\|<$ 1 , on $D_{k}$. Choose an ordered orthonormal basis for $T_{p} D_{k} \cap\left(E_{p}^{c} \oplus E_{p}^{u}\right)$ and $T_{p} D_{k} \cap$ $\left(E_{p}^{c} \oplus E_{p}^{s}\right)$ and use an affine coordinate change that takes $p$ to 0 and these bases 
to the standard bases for $\mathbf{R}^{u} \oplus 0$ and $0 \oplus \mathbf{R}^{s}$. When $t_{0}>0$ is chosen with $\phi_{t_{0}}(p) \in$ $\operatorname{int}\left(D_{k^{\prime}}\right)$ and $p^{\prime} \in D_{k^{\prime}}$ is near $\phi_{t_{0}}(p)$ we choose coordinates in this same way on $D_{k^{\prime}}$. Let $r>0$ be a constant small relative to the distances from $p$ to the boundary of $D_{k}$. If $p^{\prime}$ is sufficiently near $\phi_{t_{0}}(p)$, the return map for $\phi_{t}$ gives a hyperbolic correspondence in local coordinates on the product $B_{r}^{u} \times B_{r}^{s}$ of balls of radius $r$, more precisely from $B_{r}^{u}(p) \times B_{r}^{s}(p)$ to $B_{r}^{u}\left(p^{\prime}\right) \times B_{r}^{s}\left(p^{\prime}\right)$. We can arrange that the major constants of this correspondence are $<\sigma$ and the minor constants are smaller than any given positive $\rho$, by choosing $r$ small enough and $p^{\prime}$ near enough to $\phi_{t_{0}} p$.

Now we use [B1] to find a fine Markov family of section $R_{j}$ with each $R_{j} \subset$ $\Omega \cap \operatorname{int}\left(D_{k}\right)$ for some $k=k(j)$. If the $R_{j}$ are sufficiently small then for any choice of $p_{j} \in R_{j}$ and any Markov transition $i$ from $\alpha(i)$ to $\omega(i)$, the Markov correspondence from $R_{\alpha(i)}$ to $R_{\omega(i)}$ extends to a hyperbolic correspondence $f_{i}$ from $X_{\alpha(i)} \times Y_{\alpha(i)}$ to $X_{\omega(i)} \times Y_{\omega(i)}$ with $f_{i}=f_{p, t_{0}}$ for $p=p_{\alpha(i)}$ and $X_{j}=B_{r}^{u}\left(p_{J}\right), Y_{j}=B_{r}^{s}\left(p_{J}\right)$. We arrange that the major constants for the cross maps $c_{i}$ are $<\sigma$ and the minor constants are $<\rho$, where $\rho$ is the positive function of $\sigma$ given in Proposition 1 .

Next we associate a system of weights $r_{i}$ to our lift $\psi_{t}$. Fix $i$ and choose $x^{\prime} \in X_{\omega(l)}$ and $y \in Y_{\alpha(i)}$ and let $\left(x, y^{\prime}\right)=c_{l}\left(x^{\prime}, y\right)$. Then $\left(x^{\prime}, y^{\prime}\right)=\phi_{t}(x, y)$ for $t=t_{i}\left(x^{\prime}, y\right)$ a positive $C^{\omega}$ function, the return time for the $i^{\text {th }}$ transition. Then $\psi_{t}: \xi_{(x, y)} \rightarrow \xi_{\left(x^{\prime}, y^{\prime}\right)}$ is represented, using our trivialization of $\xi \mid\left(\cup_{k} D_{k}\right)$, by an invertible $d \times d$ matrix $r_{i}\left(x^{\prime}, y\right)$.

Thus our $C^{\omega}$ product neighborhoods $X_{j} \times Y_{j}$ of the Markov section $\mathbf{R}_{j}$ and our $C^{\omega}$ trivializations of $\xi \mid D_{k}$ define a system of $C^{\omega}$ hyperbolic correspondences $f_{l}$ and $C^{\omega}$ weights $r_{l}$, indexed by the Markov transitions. As in Sect. 5, this system defines a transfer operator $L$. Provided $\psi_{t}$ is sufficiently contractive as $t \rightarrow+\infty$, so that the series in $\zeta(\psi)$ coverges absolutely and $\|L\|<1$, we will compare $\zeta(\psi)$ to $\operatorname{det}(I-L)$.

Suppose $\vec{i}=\left(i_{1}, \ldots, i_{n}\right) \in \Lambda_{n}$ is an $n$-loop with multiplicity $\mu(\vec{i})$. There are $n / \mu(\vec{i})$ distinct elements of $\Lambda_{n}$ obtained by cyclically permuting $\vec{i}$ and altogether these contribute a factor

$$
\exp -\left[\frac{1}{\mu(\vec{i})} \frac{(-1)^{u} \operatorname{Tr}\left(t_{1} \circ \cdots \circ t_{n}\right)(p)}{\operatorname{Det}\left(I-D_{p}\left(F_{l_{n}} \circ \cdots \circ F_{l_{1}}\right)\right)}\right] .
$$

Since $F_{i_{n}} \circ \cdots \circ F_{i_{1}}$ has a fixed point, $p$ is real and

$$
\operatorname{Det}\left(I-D_{p}\left(F_{l_{n}} \circ \cdots \circ F_{i_{1}}\right)\right)=\operatorname{Det}\left(I-D_{p}\left(f_{i_{n}} \circ \cdots \circ f_{\iota_{1}}\right)\right)=\operatorname{Det}\left(I-\mathbf{P}_{\gamma}\right)
$$

where $\gamma$ is the periodic orbit through $p$ determined by $\vec{i}$. Moreover $\left(t_{1} \circ \cdots \circ t_{n}\right)(p)$ is the holonomy of $\psi$ around $\gamma$, so

$$
\operatorname{Tr}\left(t_{1} \circ \cdots \circ t_{n}\right)(p)=\chi_{\gamma}(\psi)
$$

The terms in $-\log \operatorname{det}(I-L)$ and $-\log \zeta(\psi)$ corresponding to $\vec{i}$ are then

$$
\frac{1}{\mu(\vec{i})} \frac{(-1)^{u} \chi_{\gamma}(\psi)}{\operatorname{Det}\left(I-\mathbf{P}_{\gamma}\right)} \text { and } \quad \frac{1}{\mu(\gamma)} \frac{\chi_{\gamma}(\psi)}{\left|\operatorname{Det}\left(I-\mathbf{P}_{\gamma}\right)\right|}
$$

Here $\mu(\vec{i})$ divides $\mu(\gamma)$ and the second factors differ a sign $(-1)^{u} \varepsilon_{\gamma}=s g n \operatorname{det} U_{\gamma}$, i.e. the holonomy of the unstable orientation line bundle $\omega$ for the flat lift. 
For simplicity we consider the case $\operatorname{dim} \Omega=1$, such as holds for a suspended horseshoe. Then we may choose the $R_{j}$ 's disjoint so that $\mu(\vec{i})=\mu(\gamma)$ for all $\vec{i}$ and $\gamma$ 's correspond 1-1 to loops modulo cyclic permutations. Then we have shown

$$
\operatorname{det}(I-L)=\zeta(\psi \otimes w)
$$

Now we vary $\psi$ over some family of lifts with a compact parameter space. We choose the complex extensions $W_{j} \times Z_{J}$ so that our transfer operator is defined for all parameter values. For a family $\psi^{z a}$ for instance, with a $C^{\omega}$, we require that the function

$$
\int_{0}^{t_{l}\left(x^{\prime}, y\right)} g\left(\phi_{s} p\right) d s, p=\left(c_{i}\left(x^{\prime}, y\right), y\right)
$$

on $X_{(\omega)(i)} \times Y_{\alpha(i)}$ extends to $W_{\omega(l)} \times Z_{\alpha(l)}$ (and we can take all of $\mathbf{C}$ for our parameter space). Then the expression det $(I-L)$ defines an analytic function of these parameters, so $\zeta(\psi \otimes w)$ is an analytic function of $\psi$. As $(\psi \otimes w) \otimes w=\psi$ we deduce that $\zeta(\psi)$ is an analytic function of $\psi$. Moreover $\zeta\left(\psi^{z a}\right)$ is an entire function of $z$. In particular $T_{\psi}^{b}(z)$ is entire. Also any zeta function of one variable for $(\Omega, \phi)$ is meromorphic.

For general $\Omega$, we follow Bowen's form of the Manning counting argument for a Markov partition ([Ma, B2]) as adapted to flows in [F1]. For $m \geqq 0$ there is a directed graph with vertex set $V_{m}$ and arrow set $A_{m}$ such that a loop in $A_{m}$ defines a periodic orbit $\gamma$ which passes at all times through at least $m+1$ Markov flowboxes. One has the inclusion-exclusion formula

$$
\frac{1}{\mu(\gamma)}=\sum_{m}(-1)^{m} \sum_{\vec{i}} \frac{\varepsilon(\vec{i})}{\mu(\vec{i})},
$$

where $\vec{i}$ runs over loops in $A_{m}$ representing $\gamma$ and $\varepsilon(\vec{i})= \pm 1$, see [F1], Proposition 1 . For $m=0, V_{0}$ and $A_{0}$ consist of the Markov vertices $j$ and transitions $i$ respectively, and $\varepsilon(\vec{i})=1$ for all $\vec{i}$. We set

$$
\zeta_{m}(\psi)=\exp -\sum_{\vec{i}} \frac{\varepsilon(\vec{i})}{\mu(\vec{i})} \frac{(-1)^{u} \chi_{\gamma}(\psi)}{\operatorname{Det}\left(I-\mathbf{P}_{\gamma}\right)},
$$

where $\vec{i}$ runs over loops in $A_{m}$ and $\psi_{t}$ is sufficiently contracting as $t \rightarrow+\infty$. Then $\zeta(\psi)$ is a finite alternating product

$$
\zeta(\psi)=\prod_{m} \zeta_{m}(\psi)^{(-1)^{m}} .
$$

As above, $\zeta_{0}(\psi \otimes w)=\operatorname{det}(I-L)$. By the construction of the $A_{m}$ and $V_{m}$ in [F1], one can easily imitate the construction of $L$ for any $m \geqq 0$ to obtain kernel transfers $L_{m}, m=0,1,2, \ldots$ with $L_{0}=L$ and

$$
\zeta_{m}(\psi \otimes w)=\operatorname{det}\left(I-L_{m}\right) .
$$

The $L_{m}$ depend linearly on $d \times d$ weight matrices that in turn depend holomorphically on $\psi_{t}$, so this formula extends $\zeta_{m}(\psi \otimes w)$ to an analytic function of $\psi$. Taking the alternating product over $m, \zeta(\psi \otimes w)$ is a meromorphic function of $\psi$. Hence $\zeta(\psi)$ is meromorphic in $\psi$, and we have the required meromorphic extensions of zeta functions of one variable. 
The weight functions for $\psi^{z}$ are $\exp \left(-z t_{i}\right) r_{l}$ with $\delta<t_{i}<1$ on $X_{\omega(l)} \times Y_{\alpha(l)}$. We may assume the $W_{j}$ and $Z_{j}$ are chosen so that $\left|t_{i}\right|<1$ on $W_{\omega(i)} \times Z_{\alpha(i)}$. Then

$$
\left\|\exp \left(-z t_{i}\right) r_{l}\right\| \leqq\left\|r_{l}\right\| e^{|z|}
$$

and Lemma 5, applied to $L_{0}, L_{1}, L_{2}, \ldots$ shows that for some positive $C$

$$
\log \left|\operatorname{det} I-L_{m}\left(\psi^{z}\right)\right| \leqq C|z|^{\operatorname{dim} M},
$$

proving the theorem.

Note that the same bound holds for $\log \left|\zeta\left(\psi^{z a}\right)\right|$, so $\zeta\left(\psi^{z a}\right)$ also has order $\leqq$ $\operatorname{dim} M$ for any $C^{\omega}$ function $a$.

We wish to correct a misstatement in [F1]. The inequality on p. 507,6 lines from the bottom should use $e^{a|z|}$ instead of $e^{-a \operatorname{Re}(z)}$. All the assertions concerning order of functions are unchanged but those concerning right order (i.e. bounds on halfplanes $\operatorname{Re} z \geqq \sigma$ ) are in doubt. Theorems 3 and 4 should be amended, with "right order" replaced by "order."

We showed above that $\zeta(\psi)$ is analytic in $\psi$ when $\operatorname{dim} \Omega=1$. This implies that $T^{b}(z)$ is expressible as a ratio of entire functions independent of the choice of Markov sections:

$$
T^{b}(z)=T_{\psi^{+}}(z) / T_{\psi^{-}}(z) .
$$

We conjecture that $\zeta(\psi)$ is analytic for $\operatorname{dim} \Omega>1$ as well as suggested by the formalism $\zeta(\psi)=\operatorname{det}_{\zeta}\left(-X_{\psi}\right)$. One approach to this would be analytic sheaf cohomology, cf [R2] and example II in Sect. 8. Another would be to develop a $C^{\infty}$ theory of kernel transfer, comparable to the results of [R2, F5] for expanding maps. For $u=s=1$ Rugh has recently proven that $\zeta\left(\psi^{z a}\right)$ is entire for $\xi$ the trivial bundle and $\psi$ the trivial lift.

\section{Section 8. Examples}

I. We first illustrate our use of generalized functions in an ad hoc example. Let $G=$ $T^{n}$ be the $n$-torus and $\hat{G}=\mathbf{Z}^{n}$. Given any positive weights $w_{\chi}, \chi \in \mathbf{Z}^{n}$, we consider the weighted Hilbert space $H\left(w_{\chi}\right)$ of Fourier series $\Sigma_{\chi} c_{\chi} \chi$ with $\Sigma\left|c_{\chi}\right|^{2} w_{\chi}<\infty$. We first let $w_{\chi}=\exp \left(2 \varepsilon \sum_{i=1}^{n}\left|\chi_{l}\right|\right)$ to obtain a space $H_{\varepsilon}$. Clearly $H_{0}=L^{2}\left(T^{n}\right)$. For $\varepsilon>0, H_{\varepsilon}$ consists of functions holomorphic on the $n$-fold product of annuli $R_{\varepsilon}=$ $\left\{\left(z_{1}, \ldots, z_{n}\right):\left|z_{i}\right|,\left|z_{i}^{-1}\right|<\exp (\varepsilon)\right\}$ with $L^{2}$ boundary values. For $\varepsilon<0, H_{\varepsilon}$ consists of generalized functions in the dual of $H_{|\varepsilon|}$.

Suppose $A: T^{n} \rightarrow T^{n}$ is a hyperbolic toral automorphism with dual automorphism $\hat{A} \in G l(n, \mathbf{Z})$. Provided $w_{\chi \circ A} / w_{\chi}$ is bounded above, $A$ acts on $H\left(w_{\chi}\right)$ by a bounded operator $A^{*}$. We introduce the splitting of $\hat{G} \otimes \mathbf{R}=\mathbf{R}^{n}=U \oplus S$ and norms on $U, S$ that are contracted by $\hat{A}^{-1}$ and $\hat{A}$, respectively. We let $H$ denote the Hilbert space $H\left(w_{\chi}\right)$ with

$$
w_{\chi}=\exp \left(\left\|\chi_{s}\right\|-\left\|\chi_{u}\right\|\right), \quad \text { for } \chi=\chi_{u}+\chi_{s}, \chi_{u} \in U, \chi_{x} \in S .
$$

If we take $\beta>1$ with $\|\hat{A} \mid S\| \leqq 1-\log \beta$ and $\left\|\hat{A}^{-1} \mid U\right\| \leqq(1+\log \beta)^{-1}$, then with $\|\chi\|=\left\|\chi_{u}\right\|+\left\|\chi_{s}\right\|$

$$
w_{\chi \circ A} / w_{\chi} \leqq \beta^{-\|\chi\|}
$$


It follows that for the orthogonal projection $\pi_{r}$ to $\chi$ 's with $\|\chi\| \leqq r, \pi_{r}: H \rightarrow H$,

$$
\left\|A^{*}-A^{*} \pi_{r}\right\|=O\left(\beta^{-r}\right) .
$$

Since rank $\pi_{r}=O\left(r^{n}\right)$ we see that $A^{*}$ is an $n$-compact operator on $H$.

For some $\varepsilon>0, H_{\varepsilon} \subset H \subset H_{-\varepsilon}$. However $H$ is neither a space of analytic functions nor the dual of such a space but rather some mixture of the two.

Considering the action of $A^{*}$ on $x$ 's, it is clear that $\operatorname{Tr}\left(A^{*}\right)=1$ and the only nonzero eigenfunctions of $A^{*}$ are constants.

II. Suppose $n=2$ and $\hat{A}=\left(\begin{array}{ll}0 & 1 \\ 1 & 1\end{array}\right)$ is the Fibonacci automorphism of $\mathbf{Z}^{2}$. Then $A: T^{2} \rightarrow T^{2}$ admits a Markov partition with 2 rectangles [AW], which we parametrize by $R_{1}=[0,1]^{2}$ and $R_{\rho}=[0, \rho] \times[-\rho, 0], \rho=\frac{\sqrt{5}-1}{2}$ such that the Markov correspondences are

$$
\begin{aligned}
& f_{1 \rho}(x, y)=\left(\rho^{-1} x,-\rho y\right) \text { from } R_{1} \text { to } R_{\rho}, 0 \leqq x \leqq \rho^{2}, 0 \leqq y \leqq 1, \\
& f_{\rho 1}(x, y)=\left(\rho^{-1} x,-\rho y\right) \text { from } R_{\rho} \text { to } R_{1},
\end{aligned}
$$

and

$$
f_{11}(x, y)=\left(\rho^{-1} x-\rho, 1-\rho y\right) \text { from } R_{1} \text { to } R_{1}, \rho^{2} \leqq x \leqq 1,0 \leqq y \leqq 1 .
$$

These correspondences are $C^{(1)}$ and split. Their cross maps $c_{1 \rho}, c_{\rho 1}, c_{11}$ are affine maps so we will ignore the choice of $W_{j}$ and $Z_{j}, j=1, \rho$. With $L=L_{1 \rho}+L_{\rho 1}+L_{11}$ as in Sect. 5 we find

$$
\operatorname{Tr} L^{q}=N_{q} \frac{-1}{\left(1-(-\rho)^{q}\right)\left(1-\rho^{-q}\right)},
$$

where $N_{q}$ is the number of period $q$ points of the Markov correspondence. Since $\hat{A}$ is the transition matrix for this correspondence, $N_{q}=\operatorname{Tr}\left(\hat{A}^{q}\right)=\rho^{-q}+(-\rho)^{q}$. Thus

$$
\operatorname{Tr} L^{q}=\frac{1+\left(-\rho^{2}\right)^{q}}{\left(1-(-\rho)^{q}\right)\left(1-\rho^{q}\right)}=\sum_{m \geqq 0, n \geqq 0}\left(\rho^{m}(-\rho)^{n}\right)^{q}+\left(\rho^{m+1}(-\rho)^{n+1}\right)^{q} .
$$

It follows that the nonzero spectrum of $L$ consists of 2 double sequences

$$
\rho^{m}(-\rho)^{n} \quad \text { and } \quad \rho^{m+1}(-\rho)^{n+1}, \quad(m, n) \geqq(0,0) .
$$

This can be seen more concretely as follows. Suppose $f(x, y)$ is an eigenkernel for this transfer with eigenvalue $\lambda$. Then $\frac{\partial f}{\partial x}$ and $\frac{\partial f}{\partial y}$ (if they are nonzero) are eigenkernels with eigenvalues $\rho \lambda$ and $(-1 / \rho) \lambda$. By inspection, $L$ fixes the kernel $\alpha$ with $\alpha_{j}(x, y)=1,0 \leqq x \leqq j,=0$ for other $x(j=1, \rho)$. The first double sequence, then, consists of generalized functions which are polynomial of degree $n$ in $y$ whose $n^{\text {th }} y$ derivative is $\left(\frac{\partial}{\partial x}\right)^{m} \alpha$. Note that $\frac{\partial x}{\partial x}$ is a delta function at $x=0$ minus a delta function at $x=j$, for $j=1, \rho$.

The second double sequence has a similar interpretation starting from an eigenkernel $\beta$ with eigenvalue $-\rho^{2}$. Here too, $\beta$ depends only on $x$ and is supported on $[0,1] \coprod[0, \rho]$. This can be seen by considering the hyperbolic correspondence on this pair of intervals given by the first coordinate of $f_{1 \rho}, f_{\rho 1}$ and $f_{11}$. For this correspondence, $u=1, s=0$, and

$$
\operatorname{Tr} L^{q}=N_{q} \frac{-1}{1-\rho^{-q}}=\left(1+\rho^{q}+\rho^{2 q}+\cdots\right)\left(1+\left(-\rho^{2}\right)^{q}\right),
$$


so the spectrum is $\rho^{m},\left(-\rho^{2}\right) \rho^{m}$. This corresponds to the $m^{\text {th }}$ derivatives of $\alpha$ and $\beta$.

Unlike $\alpha$, there is no simple formula for the generalized function $\beta(x)$. The component $\beta_{1}=\beta \mid[0,1]$ satisfies a simple functional equation that characterizes it (and hence $\beta_{\rho}=\beta \mid[0, \rho]$ ) uniquely up to a constant factor:

$$
-\rho^{2} \beta_{1}(x)=-\rho^{-2} \beta_{1}\left(\rho^{-2} x\right)+\beta_{1}\left(-\rho+\rho^{-1} x\right) .
$$

Using the results of [R2] (see also [F5]) one can see that $\beta(x)$ is in the dual of $C^{2+\varepsilon}$, for any $\varepsilon>0$.

Consider the time 1 suspension flow $\phi_{t}$ of $A$ and its zeta function $T^{b}(z)$ for the trivial bundle and lift:

$$
T^{\mathrm{b}}(z)=\exp -\sum_{q=1}^{\infty} \frac{1}{q} \tilde{N}_{q} \frac{1}{\left(1-(-\rho)^{q}\right)\left(\rho^{-q}-1\right)} e^{-z q},
$$

where $\tilde{N}_{q}=\# \operatorname{Fix}\left(A^{q}\right)$. By the Lefschetz fixed point formula, or an elementary count,

$$
\begin{aligned}
-\tilde{N}_{q} & =1-\operatorname{Tr} \hat{A}^{q}+(-1)^{q}=\left(1-(-\rho)^{q}\right)\left(1-\rho^{-q}\right), \\
T^{b}(z) & =\exp -\sum_{q=1}^{\infty} \frac{1}{q} e^{-z q}=1-e^{-z} .
\end{aligned}
$$

Thus the transfer operators $L_{0}(z), L_{1}(z), \ldots$ satisfy

$$
\prod_{m \geqq 0} \operatorname{det}\left(I-L_{m}(z)\right)^{(-1)^{m}}=1-e^{-z}
$$

This example suggests we interpret the alternating product formula for $T^{b}(z)$ in terms of a sort of analytic sheaf cohomology. We see that $L$ has a spectrum consisting of 2 double sequences, but only the eigenkernel $\alpha$ for the eigenvalue 1 gives rise to an $A$-invariant kernel on $T^{2}$. The latter is just the constant function 1 on $T^{2}$, as in example I. All the other eigenkernels for $L$ are either concentrated on the unstable boundary of $R_{1}$ and $R_{\rho}$ and project to zero in $T^{2}$ or are incompatible along the stable boundary, and do not fit together to form an eigenkernel on $T^{2}$. The sheafs involved here are based on morphisms that mix restriction and extension, like the operator $\varepsilon \rho$ of Sect. 2.

\section{Section 9. The Explicit Formula for a $C^{\omega}$ Basic Set}

Let $\mu^{\phi}$ be the atomic measure on $(0, \infty)$ supported on the length spectrum of $\phi \mid \Omega, \mu^{\phi}=\sum_{\gamma} \frac{1}{\mu(\gamma)} \delta(x-\ell(\gamma))$. We call $\mu^{\phi}$ the length distribution of $\phi \mid \Omega$. Then if $N(T)$ is the number of closed orbits of length $\leqq T$ and $N_{p}(T)$ is the number of prime closed orbits of length $\leqq T$ we can compute any of $\mu^{\phi}, N$ and $N_{p}$ using the formulas

$$
\begin{aligned}
\mu^{\phi}(0, T] & =N_{p}(T)+\frac{1}{2} N_{p}(T / 2)+\frac{1}{3} N_{p}(T / 3)+\cdots, \\
N(T) & =N_{p}(T)+N_{p}(T / 2)+N_{p}(T / 3)+\cdots .
\end{aligned}
$$


The sharpest asymptotic statements concerning the length spectrum involve certain means of $\mu^{\phi}$. Define $M_{i}(T)$ for $i \geqq 0, T>1$ inductively by

$$
M_{0}(T)=\int_{0}^{\ln T} t d \mu^{\phi}(t)=\sum_{\gamma: \ell(\gamma) \leqq \ln T} \ell(\gamma) / \mu(\gamma), M_{i+1}(T)=\int_{1}^{T} M_{i}(t) d t .
$$

By the remarks following the proof of Theorem 5 in [F2], we obtain an analogue of the Weil explicit formula for prime numbers, valid for any basic set $\Omega$ for a $C^{(1)}$ flow on a smooth manifold $M$. Take $k \geqq \operatorname{dim} M$ and $T>1$. Then $M_{k}(T)$ is a "power series" with complex coefficients and complex exponents:

$$
T^{-k} M_{k}(T)=\sum_{\rho} c_{\rho} T^{\rho}
$$

Here either $\rho \in\{0,-1, \ldots,-k\}$ and $c_{\rho}$ depends on the first 2 terms in the Laurent expansion of $R^{\prime}(s) / R(s)$ or $\rho$ is a zero/pole of $R(s)$ of order $n_{\rho}$ and $c_{\rho}=n_{\rho} / \rho(\rho+1) \cdots(\rho+k)$. We have the following direct expression for $M_{k}(T)$ in terms of periodic orbits $\gamma$ :

$$
T^{-k} M_{k}(T)=\sum_{\gamma} \frac{\ell(\gamma)}{\mu(\gamma)}\left(1-T^{-1} e^{\ell(\gamma)}\right)_{+}^{k},
$$

where $x_{+}^{k}$ is $x^{k}$ for $x \geqq 0$ and 0 for $x<0$.

For the geodesic flow over a closed hyperbolic surface, this formula was proven for $k \geqq 2$ by Randol using the Selberg trace formula [Ra]. In case $E^{u}$ is $C^{\omega}$ one can choose any $k \geqq 1+\operatorname{dim} E^{u}[\mathrm{~F} 2]$. Also similar expansions hold for $\mu^{\phi}$ replaced by $\mu^{\psi}=\sum_{\gamma} \frac{1}{\mu(\gamma)} \chi_{\gamma}(\psi) \delta(x-\ell(\gamma))$ for any $C^{\omega}$ lift $\psi$.

From knowledge of the divisor of $R(z)$ one can convert the exact formula to an asymptotic formula. Suppose $\rho_{1}, \ldots, \rho_{N}$ are the only zeroes/poles of $R(z)$ with $\operatorname{Re} z>a$ for some $a>0$. Then for any $b>a$,

$$
T^{-k} M_{k}(T)=c_{\rho_{1}} T^{\rho_{1}}+\cdots+c_{\rho_{N}} T^{\rho_{N}}+O\left(T^{b}\right) .
$$

Consider for instance a closed oriented hyperbolic manifold $X$ of dimension $d$, with unit sphere bundle $M=\Omega$ and geodesic flow $\phi_{t}: M \rightarrow M$. As $E^{u}$ is analytic and $\operatorname{dim} E^{u}=d-1$, we may take $k \geqq d$. By [F6]

$$
R(z)=\prod_{j=0}^{d-1} S_{j}(j+z)^{(-1)^{j}}
$$

where $S_{J}$ is a certain Selberg function. Thus

$$
n_{\rho}=\sum_{j=0}^{d-1}(-1)^{j} \operatorname{ord}_{\rho+j}\left(S_{j}\right)
$$

For $w$ with $\operatorname{Re} w>0, \operatorname{ord}_{w} S_{j}$ is the multiplicity of the eigenvalue $\lambda$ for the Laplacian $\Delta_{j}$ on coclosed $j$-forms, where $\lambda=(n-j)^{2}-(w-n)^{2}, n=\frac{d-1}{2}$ ([F6], p. 538). Thus an eigenvalue of $\Delta_{j}$ of the form $\lambda=\rho(d-1-2 j-\rho)$ contributes $(-1)^{J}$ to $n_{\rho}$ for $\operatorname{Re} \rho>0$. For $\operatorname{Re} \rho>n$, we use $\lambda \geqq 0$ to find that $\rho \in \mathbf{R}$ and $\rho \leqq d-1-2 j$.

Say $d \geqq 6$. It follows that if $j=1, \lambda=0$ (corresponding to a harmonic one form on $X$ ) there is a pole of $R(z)$ at $z=d-3$ (unless it is canceled by an eigenfunction 
of $\Delta_{0}$ with $\left.\lambda=2(d-3)\right)$. Thus the cohomology of $X$ affects the coefficient of $T^{d-3}$ in the asymptotic behavior of the averaged length distribution $M_{d}(T)$.

\section{Section 10. Lefschetz Formulas for Smale Flows}

This section uses our analytic continuation result to extend results of [F3], to which we refer for a more extended discussion of the notions that follow. Suppose $M$ is a closed $C^{\omega}$ manifold and $\phi_{t}: M \rightarrow M$ a $C^{\omega}$ flow such that the chain recurrent set $\Omega$ is hyperbolic. If $\operatorname{dim} \Omega=1$ we say $\phi_{t}$ is a Smale flow (this is a more general definition than that first given by Zeeman, who required the strong transversality property). Then $\Omega$ is isolated and so admits an isolating block $N . N$ is a manifold with corners whose boundary is the union of 2 manifolds with boundary $N_{-}, N_{+}$ that meet transversely in their common boundary: $N_{-} \cap N_{+}=\partial N_{-}=\partial N_{+}, N_{-} \cup$ $N_{+}=\partial N$. The flow $\phi_{t}$ is transverse to $N_{ \pm}$(inward on $N_{+}$, outward on $N_{-}$) and $\cup_{t=-\infty}^{\infty} \phi_{t} N=\Omega$. The simple homotopy type of the pair $\left.N, N_{-}\right)$is an invariant of $\phi$.

Suppose $E$ is a flat bundle over $M$ whose holonomy $\alpha$ has $|\operatorname{det} \alpha|=1$ and such that $H^{*}\left(N, N_{-} ; E\right)=0$. It follows that $H^{*}(M ; E)=0$ and that the Reidemeister torsions are equal: $\tau_{E}(M)=\tau_{E}\left(N, N^{\prime}\right)$. The latter may be calculated by choosing a cross-section $K$ for $\phi \mid N$, with return map $r: K \cup N_{-} \rightarrow K \cup N_{-}$, where $r$ fixes $N_{-}$ and $r(x), x \in K-N_{-}$, is the first point of $K \cup N_{-}$on the forward trajectory from $x$. Let $r_{i}^{*}$ be the action of $r$ on $H^{l}\left(K \cup N_{-}, N_{-} ; E\right)$ and define the Lefschetz zeta function of $(\phi \mid \Omega, E)$ as

$$
\tilde{\zeta}_{E}(t)=\prod_{i \geqq 0}\left(\operatorname{det}\left(I-t r_{i}^{*}\right)\right)^{(-1)^{i+1}}
$$

Then $\tilde{\zeta}_{E}$ is a rational function. Following Milnor's calculation of the torsion of an infinite cyclic covering, one shows $\left|\tilde{\zeta}_{E}(1)\right|^{-1}=\tau_{E}\left(N, N_{-}\right)$([F3], Theorem 3.3).

To describe $\tilde{\zeta}_{E}$ in terms of periodic orbits, we choose a Markov family of sections whose union is $K \cap \Omega$. We set

$$
Z_{E, K}(z, s)=\exp -\sum_{\gamma} \frac{1}{\mu(\gamma)} \varepsilon_{\gamma} \chi_{\gamma}\left(\psi_{E}\right) e^{-z \ell(\gamma)-s n(\gamma)},
$$

where $\psi_{E}$ denotes the flat lift of $\phi$ to $E$ and $n(\gamma)$ is the number of $t, 0<t \leqq$ $\ell(\gamma)$, where $\phi_{t} p \in K$ for $p$ in $\gamma$. Clearly $Z_{E, K}(z, s)$ is defined for $\operatorname{Re} s \geqq 0$, $\operatorname{Re} z \gg 0$ or for $\operatorname{Re} z \geqq 0, \operatorname{Re} s \gg 0$. Then Sect. 7 gives that $Z_{E, K}(z, s)$ is an entire function of $(z, s) \in \mathbf{C}^{2}$. Here we deform $\psi_{E}$ to $\psi_{E}^{z+s a}$, where $a>0$ is chosen so that $\int_{0}^{t(x)} a(\phi(x, u)) d u=1$ for $x \in K \cap \Omega$ and $t(x)$ the first return time of $x$. Then

$$
Z_{E, K}(0, s)=\tilde{\zeta}_{E}\left(e^{-s}\right)^{-1}
$$

by the Lefschetz fixed point formula with coefficients in $E$ for the iterates of $r$. Also

$$
Z_{E, K}(z, 0)=Z_{\alpha}(z)
$$

is the torsion function of $\phi \mid \Omega$ for the flat bundle $E$ with holonomy $\alpha$. We find

$$
\left|Z_{\alpha}(0)\right|=\left|\tilde{\zeta}_{E}(1)\right|^{-1}=\tau_{E}(M) .
$$


Thus $R$-torsion is obtained from the periodic orbits of $\phi$ using the Lefschetz formula. We summarize this result as follows:

Theorem 2. If $\phi$ is a $C^{\omega}$ Smale flow on $M$ and $E$ is a flat bundle on $M$ which is acyclic for a pair $\left(N, N_{-}\right)$, with $N$ an isolating block for the chain recurrent set of $\phi$, then $\phi$ is Lefschetz at $E$, that is $\left|Z_{E}(0)\right|=\tau_{E}(M)$.

The Fuller index of $\gamma$ is $\operatorname{ind}_{F}(\gamma)=\frac{1}{\mu(\gamma)} \varepsilon \gamma$. If $\alpha$ denotes the holonomy of $E$ then the preceding formula may be paraphrased

$$
\sum_{\gamma} \operatorname{ind}_{F}(\gamma) \operatorname{Tr} \alpha(\gamma)=-\log \tau_{E}(M)
$$

where the divergent series on the left is regularized by the analytic continuation result of Sect. 7.

\section{References}

[AW] Adler, R., Weiss, B.: Similarity of automorphisms of the torus. Memoirs AMS 98, (1970)

[AB] Atiyah, M., Bott, R.: Notes on the Lefschetz fixed point theorem for elliptic complexes. Harvard, 1964

[B] Bieberbach, L.: Lehrbuch der Funktionentheorie. Teubner, B.G., 1927

[B1] Bowen, R.: Symbolic dynamics for hyperbolic flows. Am. J. Math. 95, 429-460 (1973)

[B2] Bowen, R.: On Axiom A diffeomorphisms. CBMS Reg. Conf. 35, Providence, RI: AMS, 1978

[BL] Bowen, R., Lanford, O.: Zeta functions of restrictions of the shift transformation. In: Global Analysis, Proceedings of 1968 Berkeley conference. Proc. Symp. Pure Math. XIV, Providence, RI: AMS, 1970

[F1] Fried, D.: Zeta functions of Ruelle and Selberg I. Ann. Sci. E.N.S. 19, 491-517 (1986)

[F2] Fried, D.: Symbolic dynamics for triangle groups. To appear in Inv. Math.

[F3] Fried, D.: Lefschetz formulas for flows. In: The Lefschetz Centennial Conference Part III, Proceedings of 1984 conference, in Mexico City, Contemporary Mathematics 58, III (1987), pp. 19-69

[F4] Fried, D.: Counting circles. In: Dynamical Systems, Proceedings of 1986-87 Special Year conference at Univ. of Maryland. Springer LNM 1342, 196-215 (1988)

[F5] Fried, D.: Flat-trace asymptotics of a uniform system of contractions. To appear in: Erg. Th. and Dyn. Syst.

[F6] Fried, D.: Analytic torsion and closed geodesics on hyperbolic manifolds. Inv. Math. 84, 523-540 (1986)

[F7] Fried, D.: Fuchsian groups and Reidemeister torsion. In: The Selberg Trace Formula and Related Topics, Proceedings of the 1984 conference at Bowdoin College. Contemp. Math. 53, 141-163 (1986)

[F8] Fried, D.: Torsion and closed geodesics on complex hyperbolic manifolds. Inv. Math. 91, 31-51 (1988)

[Ga] Gallavotti, G.: Funzioni zeta ed insiemi basilas. Accad. Lincei, Rend. Sc. fismat. e nat. 61, 309-317 (1976)

[G] Grothendieck, A.: La theorie de Fredholm. Bull. Soc. Math. France 84, 319-384 (1956)

[GS] Guillemin, V., Sternberg, S.: Geometric Asymptotics. AMS Math Surveys 14, 1978

[Gu] Guillemin, V.: Lectures on the spectral theory of elliptic operators. Duke Math. J. 44, 485-517 (1977)

[H] Hormander, L.: An Introduction to Complex Analysis in Several Variables. Amsterdam: North-Holland, 1973

[K] Kitaev, A. Yu.: Fredholm determinants for hyperbolic diffeomorphisms of finite smoothness. Preprint

[Ma] Manning, A.: Axiom A diffeomorphisms have rational zeta functions. Bull. London Math. Soc. 3, 215-220 (1971) 
[M] Millson, J.: Closed geodesics and the $\eta$-invariant. Annals of Math. 108, 1-39 (1978)

[MS] Stanton, R., Moscovici, H.: R-torsion and zeta functions for locally symmetric manifolds. Inv. Math. 105, 185-216 (1991)

[Pa] Parry, W.: Synchronisation of canonical measures for hyperbolic attractors. Commun. Math. Phys. 106, 267-275 (1986)

[PP] Parry, W., Pollicott, M.: Zeta Functions and the Periodic Orbit Structure of Hyperbolic Dynamics. Asterisque 187-188, Soc. Math. de France, 1990

[P] Pollicott, M.: Meromorphic extension for generalized zeta functions. Inv. Math. 85 (1986)

[Ra] Randol, B.: On the asymptotic distribution of closed geodesics on compact Riemann surfaces. Trans. AMS 233, 241-247 (1977)

[R1] Ruelle, D.: Zeta functions for expanding maps and Anosov flows. Inv. Math. 34, 231-242 (1976)

[R2] Ruelle, D.: An extension of the theory of Fredholm determinants. Publ. Math. I.H.E.S. 72, 311-333 (1990)

[Ru] Rugh, H.H.: The correlation spectrum for hyperbolic analytic maps. Nonlinearity 5, 1237 1263 (1992)

[Ru] Rugh, H.H.: Generalized Fredholm determinants and Selberg zeta functions for Axiom A dynamical systems. Preprint

[S] Sanchez-Mergado, H.: Lefschetz formulas for Anosov flows on 3-manifolds. Erg. Th. and Dyn. Syst. 13, 335-347 (1993)

[Se] Selberg, A.: Harmonic analysis and discontinuous groups in weakly symmetric Riemannian spaces with applications to Dirichlet series. J. Ind. Math. Soc. 20, 47-87 (1956)

[Sm] Smale, S.: Differentiable dynamical systems. BAMS 73, 747-817 (1967)

[T] Tangerman, F.: Meromorphic continuation of Ruelle zeta functions. Thesis, Boston University, 1986

[Tr] Treves, F.: Topological Vector Spaces, Distributions and Kernels. New York: Academic Press, 1967

Communicated by A. Jaffe 